\title{
Transcriptomic analysis of interstock- induced dwarfism in Sweet Persimmon (Diospyros kaki Thunb.)
}

Yanying Shen ${ }^{1,2}$, Weibing Zhuang ${ }^{3}$, Xutong Tu' ${ }^{1}$ Zhihong Gao ${ }^{1}$, Aisheng Xiong ${ }^{1}$, Xinyi Yu ${ }^{1}$, Xuehan Li' ${ }^{1}$, Feihong Li ${ }^{1}$ and Shenchun $\mathrm{Qu}^{1,2}$

\begin{abstract}
Growth monitoring indicated that the height of 'Kanshu' plants with 'Nantong-xiaofangshi' as an interstock was significantly shorter than that of 'Kanshu' plants with no interstock. A transcriptome analysis of the two graft combinations ('Kanshu'/Diospyros lotus and 'Kanshu'/'Nantong-xiaofangshi'/Diospyros lotus) was conducted to explore the dwarfing genes related to the use of the 'Nantong-xiaofangshi' interstock. Hormone levels and water conductance were also measured in these two graft combinations. The results indicated that the levels of both IAA and GA were lower in 'Kanshu' that had been grafted onto the 'Nantong-xiaofangshi' interstock than in 'Kanshu' with no interstock; additionally, the water conductance was lower in grafts with interstocks than in grafts without interstocks. The expression of AUXIAA and auxin-responsive GH3 genes was enhanced in scions grafted on the interstock and was negatively correlated with the IAA content and growth of scions. The expression of GA2OX, DELLA, and SPINDLY genes were also upregulated and associated with a decrease in the level of GA in scions grafted on the interstock. Since one of the GA2Ox unigenes was annotated as DkGA20x1 in Diospyros kaki, but was not functionally validated, a functional analysis was conducted in transgenic tobacco. Overexpression of DKGA2Ox1 in transgenic plants resulted in a dwarf phenotype that could be recovered by the exogenous application of $\mathrm{GA}_{3}$. We conclude that the 'Nantong-xiaofangshi' interstock affects the water conductance and expression of genes related to the metabolism and transduction of IAA and GA in the grafted scion and thus regulates phytohormone levels, producing dwarfing.
\end{abstract}

\section{Introduction}

Oriental or sweet persimmon (Diospyros kaki Thunb., $2 \mathrm{n}=6 \mathrm{X}=90$ ), also called Japanese persimmon or kaki, is believed to have originated in China. Because sweet persimmon is one of the tallest fruit-tree species, the management of its trees is very labor-intensive. Therefore, the identification and use of a dwarfing rootstock or interstock could be used to control tree vigor and reduce the amount of labor required ${ }^{1}$ for management. In fact, the

Correspondence: Shenchun Qu (qscnj@njau.edu.cn)

${ }^{1}$ College of Horticulture, Nanjing Agricultural University, 210095 Nanjing, Jiangsu, China

${ }^{2}$ Jiangsu Key Laboratory for Horticultural Crop Genetic Improvement, 210014 Nanjing, China

Full list of author information is available at the end of the article. use of dwarfing rootstocks or interstocks is the primary approach for producing dwarfed fruit trees because these rootstocks and interstocks result in reduced tree volume, height, canopy diameter, and circumference ${ }^{2}$. Some persimmon cultivars that have potential dwarfing effects on grafted scions could be used as rootstocks or interstocks ${ }^{1,3}$. The dwarfed persimmons obtained by the use of dwarfing rootstocks or interstocks can be used to establish orchard systems that are easier to manage than systems without dwarfed persimmons, thus reducing labor demands while maintaining a high yield efficiency ${ }^{4}$.

Studies on the mechanism of scion dwarfing have been conducted in fruit trees, such as apple ${ }^{5}$, pear $^{6}$, peach ${ }^{7}$, and citrus $^{8}$. Current hypotheses of the dwarfing mechanisms of rootstocks and interstocks that alter scion size suggest

\section{(c) The Author(s) 2019}

(c) (i) Open Access This article is licensed under a Creative Commons Attribution 4.0 International License, which permits use, sharing, adaptation, distribution and reproduction c. in any medium or format, as long as you give appropriate credit to the original author(s) and the source, provide a link to the Creative Commons license, and indicate if changes were made. The images or other third party material in this article are included in the article's Creative Commons license, unless indicated otherwise in a credit line to the material. If material is not included in the article's Creative Commons license and your intended use is not permitted by statutory regulation or exceeds the permitted use, you will need to obtain permission directly from the copyright holder. To view a copy of this license, visit http://creativecommons.org/licenses/by/4.0/. 
that hydraulic properties ${ }^{9}$, nutrients ${ }^{10}$, phenols ${ }^{11}$, enzymatic activity ${ }^{12}$, and phytohormones ${ }^{13}$ all may play a role. Some studies have indicated that the influence of these factors is dependent on the nature of the graft union anatomy $^{14}$ and resistivity ${ }^{15}$. However, some studies have reported that the degree of graft union compatibility is not affected the translocation and transport of nutrients and ions ${ }^{16,17}$. It is well known that hormones play an important role in regulating tree size. The dwarfing effect of specific rootstocks or interstocks is due to alterations in the expression of genes associated with hormone metabolism and transduction, which in turn may regulate the balance of endogenous hormones in scions ${ }^{18,19}$.

Rootstocks can influence the metabolism and transport of IAA in grafted scions. Early studies found that the basipolar transport of IAA in dwarfing rootstocks was lower than that in semidwarfing rootstocks ${ }^{20}$. Lochard and Schneider ${ }^{15}$ postulated that a dwarfing rootstock could affect the basipetal transport of IAA in the phloem and cambium of the rootstock stem, resulting in a decreased supply of IAA to roots ${ }^{11}$. GA is also related to plant growth and development and plays a direct role in stem elongation growth ${ }^{21}$. Previous research provided evidence that GAs can exhibit nonpolar transport over long distances in grafted plants and affect growth ${ }^{22}$. Hooijdonk et al. ${ }^{23}$ suggested that the transmission of GAs is suppressed by the reduction in the basipetal transport of IAA to the root and that this might decrease the duration of elongation growth. Moreover, root-produced $\mathrm{GA}_{19}$ supplied to the scion, a potential precursor of bioactive $G_{1}$, which is required for shoot extension, is restricted by dwarfing rootstocks ${ }^{24}$. Interstocks can also effectively govern the growth of grafted plants ${ }^{25}$. A study examining the dwarfing mechanism of interstocks indicated that interstocks can affect the levels of some hormones and gene expression in the stem ${ }^{26}$. Persimmons grafted onto a dwarfing interstock can effectively control scion growth ${ }^{27}$; however the mechanism responsible for the dwarfing effect of interstocks is still not clearly understood.

Many studies have demonstrated that grafting can alter gene expression in scions. Jensen et al. ${ }^{5}$ reported the gene expression patterns in 'Gala' apple scions grafted onto different rootstocks and identified genes potentially associated with horticulturally important traits. Prassinos et al. $^{28}$ found that the timing of differentially expressed genes (DEGs) coincided with the early cessation of terminal shoot growth in a scion/rootstock combination and suggested that these DEGs might be involved in the dwarfing phenomenon. Vascular tissues theoretically provide opportunities for the long-distance translocation of micromolecules and macromolecules ${ }^{29}$. The presence of mobile mRNA in the phloem was first reported in potato $^{30}$. Subsequently, a series of transported mRNA studies in phloem tissues were conducted in a variety of species, including Arabidopsis ${ }^{31}$, pumpkin ${ }^{32}$, pear ${ }^{33}$, and apple $^{34}$. Other studies have demonstrated through grafting experiments that certain mRNAs can be transported in the vascular system based on a noncellular autonomous pathway mechanism ${ }^{35}$. Additional studies documented the translocation of endogenous mRNAs, including $G A I^{36,37}, A U X / I A A 14^{34}, K_{N O T T E D 1^{38}}, N A C^{39}$, and $S t B E L 5^{40}$, where GAI, AUX/IAA14, and NAC mRNAs exhibited bidirectional transport in grafted plants. Collectively, these studies have demonstrated that mRNAs are not only transported across cellular interfaces but also function as long-distance signaling molecules and thus may play a significant role in the regulation of plant physiology and development ${ }^{34,41}$. Grafting experiments have also confirmed that the rootstock-scion transport of mutated gene mRNA induces phenotypic changes ${ }^{42,43}$.

As recorded in 1982 in the 'Fruit Tree Resources Survey of Jiangsu Province', Nantong City (Rudong district and Hai'an district) China, 'Nantong-xiaofangshi' persimmon (Diospyros kaki Linn. cv. Nantong-xiaofangshi) exhibits many advantageous characteristics. These characteristics include significant dwarfing, robust growth, high adaptability, early bearing, high and stable yield, excellent fruit quality, and astringency that is relatively easy to remove ${ }^{44}$. Although the dwarfing ability of 'Nantong-xiaofangshi' persimmon is much greater than that of the two persimmon cultivars 'Dafangshi' and 'Zhushahong' ${ }^{15}$, the seeds of 'Nantong-xiaofangshi' are rare, and it is difficult for the cuttings and tissue culture seedlings of 'Nantongxiaofangshi' to root. However, due to its excellent horticultural traits, 'Nantong-xiaofangshi' has been used as a dwarfing interstock in persimmon, even though the mechanisms responsible for the dwarfing effect of interstocks have not yet been elucidated.

In the present study, we examined the entire vascular system of persimmon stems relative to the impact of a dwarfing interstock. RNA-seq analysis of 'Kanshu'/ Diospyros lotus and 'Kanshu'/'Nantong-xiaofangshi'/ Diospyros lotus was conducted to select candidate unigenes associated with the dwarfing effect induced by using 'Nantong-xiaofangshi' as an interstock. The results indicated that some unigenes were concentrated in hormone signal metabolism and transduction. Therefore, the levels of endogenous IAA, GA, and ABA were measured in the same tissues. In GA metabolism, GA2ox can catalyze bioactive GA and convert it to an inactive product. The functional role of GA2ox genes in dwarfing has been previously demonstrated in several species ${ }^{46,47}$. Since a unigene in Diospyros kaki was annotated as DkGA2ox1 but has not been functionally confirmed, we examined the effect of DkGA2ox1 in transgenic tobacco. We also investigated the transport of endogenous DkGA2ox1 transcripts from 'Nantong-xiaofangshi' interstock tissues 
to the scion. Our study provides molecular information that helps clarify the dwarfing mechanism associated with the use of 'Nantong-xiaofangshi' as an interstock in persimmon.

\section{Materials and methods \\ Plant material}

Taking into consideration current production practices, this study utilized nongrafted and grafted combinations of persimmon, including Diospyros lotus, 'Kanshu', 'Nantong-xiaofangshi'/Diospyros lotus, 'Kanshu'/Diospyros lotus, and 'Kanshu'/'Nantong-xiaofangshi'/Diospyros lotus ('Nantong-xiaofangshi', hereafter referred to as N.x). Seedlings with uniform growth were selected for grafting. The first grafting (rootstock grafted with interstock) was conducted in early April 2014 with 2-year-old Diospyros lotus and 1-year-old N.x used as the rootstock and interstock, respectively. The second branch grafting (interstock grafted with scion) was conducted in May 2015 with 1-year-old 'Kanshu' branches used as scions. At the same time, combinations of 'Kanshu'/Diospyros lotus and N.x/Diospyros lotus were made. The grafted 'Kanshu' persimmon plants were cultivated in soil in pots and subjected to conventional management outdoors. The experimental materials were obtained from the Introduction and Breeding Base in Kunshan (Jiangsu, China).

All persimmon stem tissues were sampled at the end of April. The tissue samples (combined phloem and xylem) were used in various analyses, and each experimental group (graft combination) contained three biological replicates that were sampled during the active growth period of persimmon. The internal tissues of the stem samples were exposed by slicing the stems with a scalpel. The collected samples were placed in $5 \mathrm{ml}$ tubes and immediately frozen in liquid nitrogen and stored at $-80^{\circ} \mathrm{C}$ for further use.

\section{Transcriptome sequencing (RNA-seq)}

Transcriptomic sequencing and analyses were conducted on stem tissues obtained from the scion $(10 \mathrm{~cm}$ above the graft union between the interstock and scion), interstock (middle of the interstock), and rootstock $(5 \mathrm{~cm}$ from the graft union) of two graft combinations, i.e., from the combination of 'Kanshu'/N.x/Diospyros lotus and the scion and rootstock from 'Kanshu'/Diospyros lotus, during the rapid elongation period (at the end of April) (Fig. 1). Before being transcriptome was sequenced, the cDNA libraries obtained from three individuals of each graft combination were separately mixed. Sequencing was performed using an Illumina HiSeq 4000 platform in paired-end mode with a read length of 150 bp by 1 GENE Science and Technology Co., Ltd. (1GENE Tech, Hangzhou, China). A total of $7.7-8.4 \mathrm{~Gb}$ of raw bases were obtained from each library. After quality assessment of

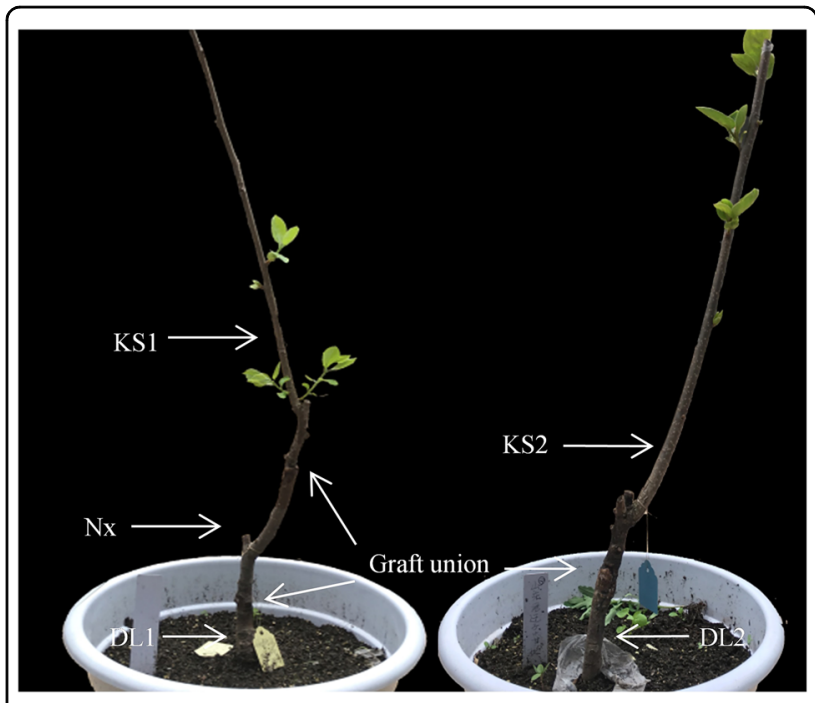

Fig. 1 Stem tissues of the two graft combinations prepared for transcriptomic sequencing and analyses. KS1, Nx, and DL1: scion, interstock and rootstock in the combination of 'Kanshu'/N.x/Diospyros lotus; KS2 and DL2: scion and rootstock in 'Kanshu'/Diospyros lotus

the obtained sequence data, adaptor sequences were removed, and raw reads with more than $20 \%$ low-quality bases (quality $<20$ ) and reads containing $>5 \%$ Ns were discarded. The remaining reads were considered clean reads. Trinity software (http://trinityrnaseq.sourceforge. net) was utilized for the de novo assembly of the transcriptome short reads ${ }^{48}$. The assembly parameters were set to 'min_contig_length $=200$, min_kmer_cov $=2$, min_glue $=3$, and seqType $=$ fq'; all other parameters set to default values. Ultimately, the contigs were clustered into unigenes with tgicl ${ }^{49}$.

The unigenes from $D$. kaki Thunb. in these two graft combinations were analyzed to select unigenes associated with dwarfing. Since the reference genome of persimmon has not yet been published, the sequences were annotated and classified by BLASTx (Version: BLAST-2.2.28) analysis with a threshold of $E$-value < $10^{-5}$ according to the NCBI nonredundant $(\mathrm{Nr})$ database (as of March, 2016), SwissProt, Clusters of Orthologous Groups (COG) and Kyoto Encyclopedia of Genes and Genomes (KEGG) $)^{50}$. Based on the Nr database annotations, Blast2 $\mathrm{GO}^{51}$ and $\mathrm{WEGO}^{52}$ were used for Gene Ontology (GO) annotation and functional classification with the default parameters. Fragments per $\mathrm{kb}$ per million fragments (FPKM) was used to avoid the bias caused by different gene lengths ${ }^{53}$.

\section{Phytohormone levels}

Phytohormones were extracted from stems of the two grafted combinations at the same sampling positions as those described in 'Transcriptome Sequencing (RNAseq)'. Three biological replicates were used for the 
determination of each hormone. The levels of gibberellin $\left(\mathrm{GA}_{4}\right)$, indole-3-acetic acid (IAA), and abscisic acid (ABA) were determined by Zoonbio Biotechnology Co., Ltd. (Nanjing, China) using a modification of the protocol described by You et al. ${ }^{54}$. Approximately $0.5 \mathrm{~g}$ of fresh tissue was ground in liquid nitrogen, and $5 \mathrm{ml}$ of extraction buffer composed of isopropanol/hydrochloric acid was added to each sample. The samples with extraction fluid were shaken at $4{ }^{\circ} \mathrm{C}$ for $30 \mathrm{~min}$. Subsequently, $10 \mathrm{ml}$ dichloromethane was added to each sample tube, and the samples were again shaken at $4{ }^{\circ} \mathrm{C}$ for $30 \mathrm{~min}$. This was followed by centrifugation at $13,000 \mathrm{rpm}$ for $5 \mathrm{~min}$ at $4{ }^{\circ} \mathrm{C}$, after which the organic phase was extracted. The obtained organic phase was dried under $\mathrm{N}_{2}$ in the dark and then dissolved in $400 \mu \mathrm{l}$ methanol $(0.1 \%$ methanoic acid) and subsequently filtered through a $0.22 \mu \mathrm{m}$ filter membrane. The purified product ( $2 \mu \mathrm{l}$ of injection volume) was then subjected to high-performance liquid chromatographytandem mass spectrometry (HPLC-MS/MS) analysis. HPLC analysis was performed using a poroshell SB-C18 (Agilent Technologies) column $(2.1 \mathrm{~mm} \times 150 \mathrm{~mm}$, $2.7 \mu \mathrm{m})$. The mass spectrometry conditions were as follows: the spray voltage was $4500 \mathrm{~V}$; the pressures of the air curtain, nebulizer, and aux gas were 15,65 , and $70 \mathrm{psi}$, respectively; and the atomizing temperature was $400{ }^{\circ} \mathrm{C}$. The hormone contents were quantitated by the standard curve method and calculated as the average concentration $\times$ volume coefficient (add the volume of the solvent)/ mass coefficient (the quality of the original sample).

\section{Hydraulic conductance}

We selected graft plants with relatively uniform growth to measure water conductance using a high-pressure flow meter (HPFM-Gen3, Dynamax Inc., Houston, TX), with reference to the method described in Tyree et $\mathrm{al}^{55}$. The stem was cut $5 \mathrm{~cm}$ below the graft union between the rootstock and interstock/scion. The stump was connected to the HPFM with a water-tight seal to measure water conduction resistance $\left(R_{\text {canopy }}\right)$. Next, the stem was cut 5 $\mathrm{cm}$ above the graft union between interstock and scion. The scion was connected to the HPFM to measure the resistance $\left(\mathrm{R}_{\text {scion }}\right)$. For comparison, 'Kanshu'/Diospyros lotus without interstock and 'Kanshu'/'Nantong-xiaofangshi'/Diospyros lotus were cut at the same position. The water conduction resistance of the grafting area was ' $R_{\text {canopy }}-R_{\text {scion }}{ }^{156}$. The water conductance and resistance were reciprocals of each other $\left(K_{h}=1 / R\right)$.

\section{Reverse transcription-quantitative PCR (RT-qPCR)}

Total RNA was extracted from samples of the scion stems of 'Kanshu' persimmon in the two different graft combinations ('Kanshu'/N.x/Diospyros lotus and 'Kanshu'/Diospyros lotus) using the Plant Total RNA Isolation Kit Plus (Foregene, Chengdu, China). The RNA quality and integrity were evaluated using a Nanodrop ND 1000 spectrophotometer (Nanodrop Technologies Inc., Delaware, USA) and agarose gel electrophoresis before the RNA was used for first-strand cDNA synthesis (TaKaRa, Dalian, China).

RT-qPCR was performed on an ABI Real-Time PCR System (Applied Biosystems, USA) using SYBR Premix Ex $\mathrm{Taq}^{\text {Tw }}$ (TaKaRa, Dalian, China). The $20 \mu$ reaction system contained $10 \mu \mathrm{l}$ SYBR Green I Mix, $1 \mu \mathrm{l}$ diluted cDNA

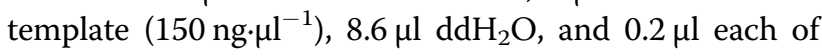
gene-specific upstream and downstream primers $(10 \mu \mathrm{M})$. The following cycling conditions were used: an initial denaturation step of $95^{\circ} \mathrm{C}(4 \mathrm{~min})$, followed by 40 cycles of $95^{\circ} \mathrm{C}(20 \mathrm{~s}), 60^{\circ} \mathrm{C}(20 \mathrm{~s})$, and $72^{\circ} \mathrm{C}(40 \mathrm{~s})$. Dissociation curves were generated from $60^{\circ} \mathrm{C}$ to $95^{\circ} \mathrm{C}$ to verify primer specificity with the presence of a single peak. Each reaction was conducted using three technical repeats and a negative control (water instead of cDNA template). All RT-qPCR reactions were carried out in accordance with the Minimum Information for Publication of Quantitative Real-Time PCR Experiments (MIQE) guidelines ${ }^{57}$. Primers for the persimmon tree were designed using Beacon Designer 7.0 (Premier Biosoft International, Palo Alto, CA, USA), and the specificity of the alignment was evaluated using BioEdit on the NCBI website, as well as our transcriptome database. Actin, $U B C, G A D P H$, and $P P 2 A$ genes served as reference genes for normalization $(\text { Table S1 })^{58}$. Expression levels were calculated using the $2^{-\Delta \Delta C t}$ method $^{59}$. Each RT-qPCR evaluation of each gene utilized three biological replicates.

\section{Gene construct and tobacco transformation}

The sense primer DkGA2ox1B-F containing a BamHI site and the antisense primer $D k G A 20 \times 1 \mathrm{~S}-\mathrm{R}$ containing a SalI site (Table 1) were utilized to create the DkGA2ox 1 mRNA plasmid construct. cDNA coding for DkGA2ox 1 was obtained from 'Nantong-xiaofangshi' using the following PCR conditions: initial denaturation at $94{ }^{\circ} \mathrm{C}$ for 4 min, followed by 35 cycles of $94^{\circ} \mathrm{C}$ for $30 \mathrm{~s}, 62^{\circ} \mathrm{C}$ for $40 \mathrm{~s}$ and $72{ }^{\circ} \mathrm{C}$ for $1 \mathrm{~min} 10 \mathrm{~s}$, and then a final extension at $72{ }^{\circ} \mathrm{C}$ for $10 \mathrm{~min}$. Amplified DkGA2ox 1 cDNA sequences were purified and subcloned into a PMD19-T simple vector (Takara, Dalian, China). The vector containing the DkGA2ox1 cDNA was digested with BamHI and SalI and then integrated into the same sites of the transformation vector, pYH4215 (Fig. S1).

The recombinant plasmid was introduced into Agrobacterium tumefaciens (strain EHA105) using the freezethaw method ${ }^{60}$ and was subsequently used for tobacco transformation. Disks of plant tissues cut from tobacco leaves were inoculated with Agrobacterium tumefaciens (strain EHA105) carrying the plasmid pYH4215DkGA2ox1 containing genes for $\beta$-glucuronidase (GUS) and hygromycin resistance (HygR) on MS medium with 
Table 1 Gene-specific primer pairs

\begin{tabular}{|c|c|}
\hline Primers & Sequences $\left(5^{\prime}-3^{\prime}\right)$ \\
\hline DKGA2OX1B-F & 5'-CGGGATCCATGGTGGTTITATC-3' \\
\hline DKGA2OX1S-R & 5'- CGAGCTCTCACGAGGCGGCAAC-3' \\
\hline DKGA2OX1-F1 & 5'-TCAAAGCCACTCTCCCACTGA-3' \\
\hline DKGA2OX1-R1 & 5'-CTCGGGAAAGTGGGGAAAACC-3' \\
\hline DKGA2OX1-S-F1 & 5'-TCTATCCCACCCGACGAAAG-3' \\
\hline DKGA2OX1-S-R1 & 5'-AGTGGGGAAAACCATCACGAG-3' \\
\hline Nt GA200x-F & 5'-TGCTTTCTTTCTTTGTCCAA-3' \\
\hline Nt GA200x- R & 5'-CTCTGTAATGCTTCTGTGTAA-3' \\
\hline Nt GA3OX - F & 5'-AAGACTGATGTGGCTCAT-3' \\
\hline Nt GA3Ox -R & 5'-TGTTAATATGGTAGAATCCGTATGT-3' \\
\hline Nt tubA1-F & 5'-CTTATGTTCCGTGGTGATG-3' \\
\hline Nt tubA1-R & 5'-TTGGTGGCTGATAGTTGA-3' \\
\hline
\end{tabular}

BA $1.0 \mathrm{mg} \cdot \mathrm{l}^{-1}$ and NAA $0.2 \mathrm{mg} \cdot \mathrm{l}^{-1}$ (pH 5.8). After 2-3 days in the dark, explants were transferred to a selective MS medium containing 6-BA $1.0 \mathrm{mg} \cdot \mathrm{l}^{-1}$, NAA $0.2 \mathrm{mg} \cdot \mathrm{l}^{-1}$, Hyg $30 \mathrm{mg} \cdot \mathrm{l}^{-1}$, and $\mathrm{Cb} 200 \mathrm{mg} \cdot \mathrm{l}^{-1}$ (pH 5.8). Next, regenerated shoots $3-5 \mathrm{~cm}$ in height were cut and cultured in $1 / 2$ MS medium containing IBA $0.2 \mathrm{mg} \cdot \mathrm{l}^{-1}$, Hyg $30 \mathrm{mg}^{-1} \mathrm{l}^{-1}$, and $\mathrm{Cb} 200 \mathrm{mg} \cdot \mathrm{l}^{-1}(\mathrm{pH}$ 5.8) to promote root development. The initial selection of DkGA2ox1 transgenic tobacco leaves was based on Hyg resistance and GUS staining.

\section{Transgenic tobacco phenotyping}

The propagation of transgenic tobacco and nontransgenic tobacco lines occurred through vegetative reproduction. The plants were grown on agar medium and transplanted to soil in pots 30 days after germination. Eighty days after their placement in the greenhouse, three wild-type plants and three plants from each transgenic line were used to measure plant height, internodal length, stem diameter, and leaf length and width. Phenotypic measurements of the transgenic plants were undertaken using two independent lines (three plants from each line). The length:width ratio of the leaves was calculated. Mature, fully expanded functional leaves of the transgenic and wild-type tobacco were collected during the blooming period for the measurement of GA levels as previously described.

\section{Expression analysis of transgenic tobacco genes}

Gene-specific primers were designed for tobacco GA20ox (Accession number AB109762) and GA3ox (Accession number AB032198) sequences registered at NCBI (Table 1). The primers were utilized to examine the expression of these genes in transgenic and nontransgenic tobacco plants. A Tubulin gene from tobacco was used as a reference gene for the normalization of expression data. The reaction system contained $10 \mu \mathrm{l}$ SYBR Green I Mix,

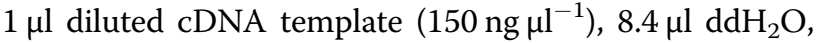
and $0.3 \mu \mathrm{l}$ each of the forward and reverse primers $(0.15$ pmol. $\left.\mathrm{l}^{-1}\right)$. The following cycling conditions were used: an initial denaturation step of $95^{\circ} \mathrm{C}(4 \mathrm{~min})$, followed by 40 cycles of $95^{\circ} \mathrm{C}(20 \mathrm{~s}), 60^{\circ} \mathrm{C}(20 \mathrm{~s})$, and $72^{\circ} \mathrm{C}(40 \mathrm{~s})$. Each expression analysis utilized three biological and technical replicates. The expression analysis was conducted utilizing ABI Real-Time PCR System software and the $2^{-\Delta \Delta C t}$ method.

\section{Cloning and sequencing of DkGA2ox 1 in different cultivars}

The open reading frame (ORF) of $D k G A 2 o x 1$ in 'Kanshu', N.x, and Diospyros lotus was cloned. Gene-specific primers for $D k G A 2 o x 1$ were designed based on sequences from Diospyros kaki Linn. cv. Nantong-xiaofangshi deposited in the NCBI database (GenBank ID, KJ664180.1) and our own RNA-seq database.

PCR-amplified cDNA fragments from Diospyros lotus, 'Nantong-xiaofangshi' and 'Kanshu' using DkGA2ox1-F1 and DkGA2ox1-R1 primers were obtained and sequenced (Table 1). Amplified cDNA sequences were purified and subcloned into the pEASY $^{\oplus}$ - Blunt cloning vector (TransGen Biotech, Beijing, China) for sequencing.

\section{Cleaved amplified polymorphic sequence (CAPS) analysis}

Restriction enzyme cleavage sites of Diospyros lotus, N. $\mathrm{x}$, and 'Kanshu' were constructed, and a specific ApaI cut site was identified (Thermo Scientific, Waltham, MA, USA). RT-PCR products were amplified with the DkGA2ox1-S-F1 and DkGA2ox1-S-R1 primers (Table 1) according to the instructions of PrimerSTAR GXL Polymerase (Takara, Dalian, China) and using the following PCR conditions: initial denaturation at $98^{\circ} \mathrm{C}$ for $5 \mathrm{~min}$, followed by 30 cycles of $98^{\circ} \mathrm{C}$ for $10 \mathrm{~s}, 60^{\circ} \mathrm{C}$ for $15 \mathrm{~s}$ and $68^{\circ} \mathrm{C}$ for $30 \mathrm{~s}$, and a final extension at $68^{\circ} \mathrm{C}$ for $10 \mathrm{~min}$. The amplified products were digested with ApaI and separated by $16 \%(\mathrm{~m} / \mathrm{v})$ polyacrylamide gel electrophoresis (PAGE).

\section{Statistical analyses}

Experimental data were subjected to analysis of variance (ANOVA) using SAS 8.0 software (SAS Institute, Inc., Cary, NC, USA). The phylogenetic analysis was performed in MEGA 6.0 software (MEGA, Tempe, AZ, USA) ${ }^{61}$. Additional data analyses were performed using Graphpad Prism 6.0 (GraphPad Software, La Jolla, CA, USA) and Microsoft Excel 2007 (Microsoft Corporation, Redmond, WA, USA). 


\section{Results}

\section{Effect of 'Nantong-xiaofangshi' on scion height}

The initial scion length was $15 \mathrm{~cm}$. After nearly one year, the scion length was measured from the top of the graft union to the shoot apex of the scion in each of the graft combinations beginning in late March 2016. The use of 'Nantong-xiaofangshi' as an interstock had a significant impact on the height of grafted trees (Fig. 2). After almost a year of growth, the shoot lengths of 'Kanshu' grafted on 'Nantong-xiaofangshi' interstocks were shorter than those grafted directly onto rootstocks. During the initial period of spring growth, the growth of 'Kanshu' scions without an interstock was significantly faster than the growth of plants grafted with an interstock. In the later stages of the growth period, after April 25, the growth of 'Kanshu' scions grafted on N.x. interstocks slowed dramatically relative to their growth on rootstocks without interstocks. There was no significant difference in growth rate between the two graft combinations after May 10.

\section{RNA-sequence analysis and functional annotation of persimmon transcripts in grafted trees}

Gene expression in grafted persimmon trees was analyzed by RNA-seq in order to better understand the mechanisms underlying interstock-induced dwarfing. Five cDNA libraries were prepared from three tissues (scions, interstocks, and rootstocks) in the 'Kanshu'/N.x/Diospyros lotus trees and from two tissues (scions and rootstocks) in the 'Kanshu'/Diospyros lotus trees. The resulting cDNA libraries were sequenced on an Illumina HiSeq 4000 platform. After stringent quality evaluation and data filtering, 6.9-7.4 Gb of clean bases were obtained from each library (Table 2). The Q20, Q30 (sequencing error rate < 0.01 ), and GC content values were 98.32-98.48\%, 95.90-96.25\%, and 47.78-48.20\%, respectively (Table 2). Contigs assembled from the high-quality reads were used to produce unigenes. Based on genetic divergence, 'Kanshu' and 'Nantong-xiaofangshi' belong to the same species (Diospyros kaki), and sequences from the tissues of the same species were assembled together. The sequence data were deposited in Figshare under https://doi.org/10.6084/ m9.figshare.7406648SRP048768. This approach was used to maximize both the number and length of the unigenes that were obtained. Only data from Diospyros kaki were used to find candidate unigenes related to interstockinduced dwarfism. The length distribution of all transcripts is shown in Fig. S2. Ultimately, a total of 119,573 unigenes with a mean length of 662 nt were obtained.

Functional annotations of unigenes were based on protein sequence similarity, KEGG pathway, COG, and Gene Ontology (GO) analyses. Unigene sequences were queried against protein databases (Nr, SwissProt, KEGG, and COG) using blastx and against the nonredundant NCBI nucleotide $(\mathrm{Nt})$ database using blastn ( $E$-value <

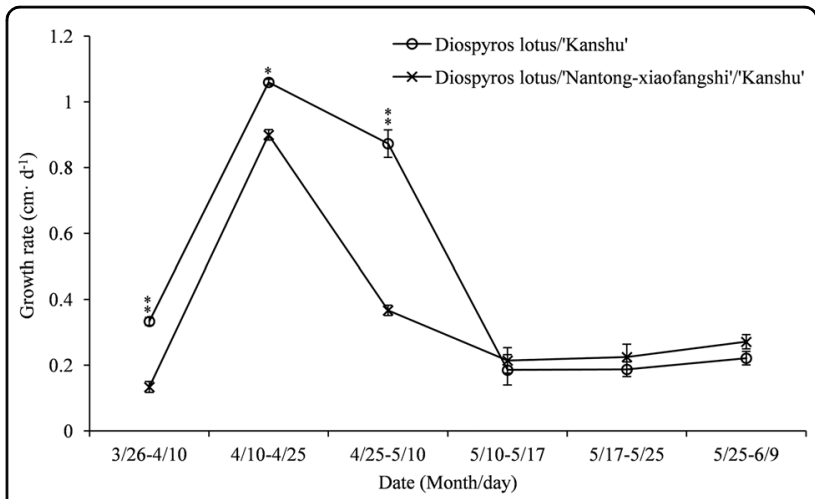

Fig. 2 Changes in the 'Kanshu' scion growth rate for different graft combinations. Error bars indicate the standard error of five biological replicates. Asterisks indicate significant differences; one asterisk represents $P<0.05$, and two asterisks represents $P<0.01$

0.00001). A total of 91,353 predicted unigenes, which comprised 85,342 (71.37\%) unigenes annotated by the $\mathrm{Nr}$ database, 60,380 (50.50\%) unigenes annotated by SwissProt, 49,032 (41.01\%) unigenes annotated by KEGG, 31,219 (26.11\%) unigenes annotated by COG, 54,925 unigenes annotated by GO terms (45.93\%), and 71,400 (59.71\%) unigenes annotated by the $\mathrm{Nt}$ database (Table S2). A homology search of the unigenes was performed using the $\mathrm{Nr}$ database, which annotated the highest number of unigenes among the six databases. According to the Nr database similarity search, a total of $45.15 \%$ of the sequences showed a high degree of similarity (E-values $<1 \mathrm{E}^{-45}$ ), and $54.85 \%$ of the sequences showed reasonable similarity (E-values within the range of $1 \mathrm{E}^{-5}$ to $\left.1 \mathrm{E}^{-45}\right)$; this result indicates a high alignment reliability (Fig. S3a). According to the similarity distribution, $30.87 \%$ of the sequences showed more than $80 \%$ similarity with sequences in the $\mathrm{Nr}$ database, indicating a good function annotation (Fig. S3b). Regarding the sources of matching sequences, Vitis vinifera provided the greatest number of blastx matches, matching $24.6 \%$ of the total number of unigenes, followed by Coffea canephora, Sesamum indicum, Theobroma cacao, Indian lotus, Citrus sinensis, and Nicotiana sylvestris (Figure S3c).

\section{GO term analysis}

GO analysis provided a functional annotation analysis of all unigenes in Diospyros kaki. Figure S4 illustrates the number of unigenes in each GO category. The GO terms for unigenes in the biological processes category included: biological regulation, cellular component organization or biogenesis, cellular, developmental, establishment of localization, growth, localization, metabolic, multicellular organismal, regulation of biological process, response to stimulus, signaling, and single-organism. In the cellular components category, unigenes were classified into cell, 
Table 2 Summary of Illumina sequencing results

\begin{tabular}{lllllll}
\hline Samples & Total raw reads & Total clean reads & Total clean bases & Q20 percentage & Q30 percentage & GC percentage \\
\hline KS2 & $56,025,676$ & $49,604,844$ & $7,440,726,600$ & $98.32 \%$ & $95.90 \%$ & $47.78 \%$ \\
KS1 & $53,887,352$ & $47,093,378$ & $7,064,006,700$ & $98.48 \%$ & $96.25 \%$ & $48.20 \%$ \\
IN & $51,690,206$ & 46443640 & $6,966,546,000$ & $98.49 \%$ & $96.27 \%$ & $47.96 \%$ \\
DL2 & $53,158,430$ & $46,435,548$ & $6,965,332,200$ & $98.56 \%$ & $96.41 \%$ & $47.98 \%$ \\
DL1 & $54,699,692$ & $48,032,432$ & $7,204,864,800$ & $98.58 \%$ & $96.45 \%$ & $48.40 \%$ \\
\hline
\end{tabular}

Note: KS1, IN and DL1: the scions, interstocks and rootstocks of 'Kanshu'/N.x/Diospyros lotus; KS2 and DL2: the scions and rootstocks of 'Kanshu'/Diospyros lotus

cell part, macromolecular complex, membrane, membrane part, organelle, and organelle part. In the molecular function category, Diospyros kaki unigenes were classified into binding, catalytic activity, enzyme regulator activity, nucleic acid binding transcription factor activity, structural molecule activity, and transporter activity. According to GO analysis, unigenes were assigned to the biological process terms of biological regulation, metabolic, developmental, signaling and growth, and the molecular function terms of catalytic and enzyme regulator activity after grafting 'Nantong-xiaofangshi' as an interstock.

\section{KEGG analysis}

KEGG pathway analysis revealed that 49,032 unigenes in Diospyros kaki were mapped to 128 predicted pathways. In the top 20 pathways with the largest number of genes, we observed that most of the unigenes were concentrated in the following pathways: metabolic pathways (ko01100), biosynthesis of secondary metabolites (ko01110), plant hormone signal transduction (ko04075), RNA transport (ko03013), phenylpropanoid biosynthesis (ko00940), and pyruvate metabolism (ko00620). Further analysis of the pathways revealed that the unigenes were also involved in peroxisome (ko04146), tryptophan metabolism (ko00380), diterpenoid biosynthesis (ko00904), zeatin biosynthesis (ko00908), and brassinosteroid biosynthesis (ko00905) pathways (Supplemental data file 1).

\section{Phytohormone levels and water conductance}

'Nantong-xiaofangshi' interstocks affected hormone levels in the grafted 'Kanshu' scions (Fig. 3). IAA levels in scions and rootstocks in the 'Kanshu'/Diospyros lotus graft combination were all significantly higher relative to their levels in the same tissues in the 'Kanshu'/N.x/Diospyros lotus graft combination. In the 'Kanshu'/N.x/Diospyros lotus trees, the IAA content was significantly higher in the interstock tissues than in the scion tissues. In contrast, no difference was observed between the interstock and rootstock tissues (Fig. 3a). GA levels in the scions of trees with 'Nantong-xiaofangshi' as an interstock were markedly lower than those in the scions of trees without an interstock. GA levels within the same grafting combination were highest in scion tissues and lowest in rootstock tissues, regardless of whether an interstock was present. This was especially apparent in 'Kanshu'/N.x/ Diospyros lotus, where the GA levels in interstock tissues were significantly lower than those in scion tissues but higher than those in rootstock tissues (Fig. 3b). For ABA levels, the results indicated that ABA levels were high in 'Kanshu'/N.x/Diospyros lotus tissues and relatively low in 'Kanshu'/Diospyros lotus tissues (Fig. 3c). In addition, the water conductance of the grafting area was significantly lower in 'Kanshu'/'Nantong-xiaofangshi'/Diospyros lotus than in 'Kanshu'/Diospyros lotus (Fig. S5).

\section{RT-qPCR analysis of genes in 'Kanshu' scions}

According to GO and KEGG analysis, the detected unigenes were related to metabolism pathways, including diterpenoid biosynthesis, tryptophan metabolism, and brassinosteroid biosynthesis pathways, and plant hormone signal transduction pathways, including gibberellin, auxin, and brassinosteroid pathways. Therefore, we analyzed candidate unigenes from these pathways in the dwarfing process with 'Nantong-xiaofangshi' as the interstock. These candidate unigenes are very likely associated with the regulation of scion growth by the interstock, and their expression levels were confirmed by RT-qPCR. The results indicated that 31 genes had significant expression differences in 'Kanshu' between 'Kanshu'/Diospyros lotus and 'Kanshu'/'Nantong-xiaofangshi'/Diospyros lotus (Fig. 4).

Regarding GA metabolism, gibberellin 2betadioxygenase (GA2ox) genes (TR24626|c0_g1_i1, TR54490|c0_g1_i1) exhibited higher levels of expression in KS1 than in KS2. Additionally, gibberellin 3betadioxygenase (GA3ox) (TR29323|c0_g1_i1) was upregulated in KS1, as was gibberellin 20-oxidase (GA20ox) (TR8775|c0_g1_i1). Regarding GA signal transduction, gibberellin insensitive dwarf 1 (GID1) gene expression was both upregulated (TR47836|c0_g1_i1, TR44942|c0_g1_i1) and downregulated (TR80297|c0_g1_i1, TR54853| c0_g1_i1) in KS1. Notably, DELLA (DELLA-family 


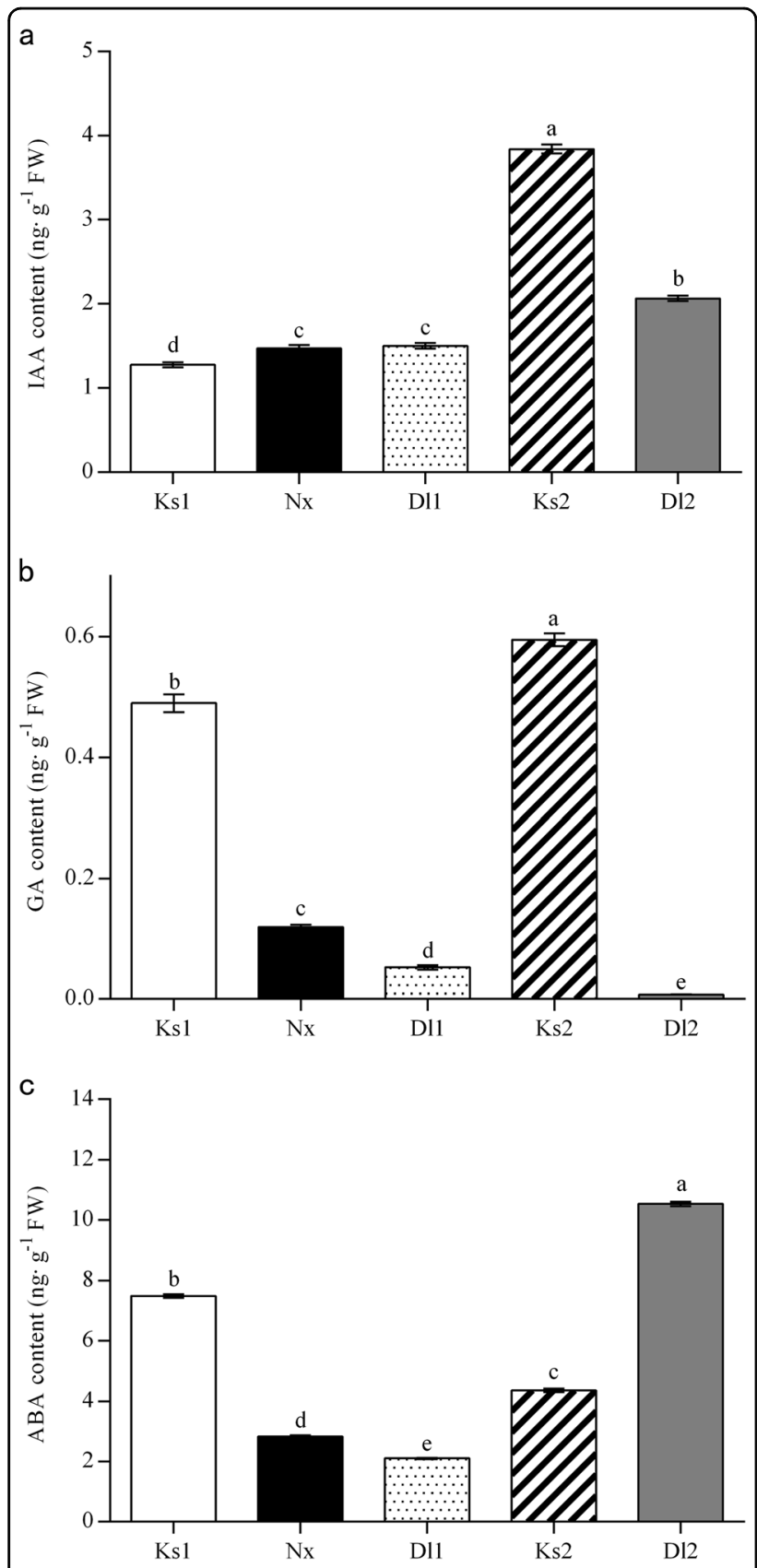

Fig. 3 Phytohormone levels in different tissues of 'Kanshu' persimmon with different graft combinations. a Indole-3-acetic acid (IAA), (b) gibberellin $\left(\mathrm{GA}_{4}\right)$, and (c) abscisic acid (ABA). Ks1, Nx, and Dl1 represent 'Kanshu', 'Nantong-xiaofangshi', and Diospyros lotus, respectively, in 'Kanshu'/'Nantong-xiaofangshi'/Diospyros lotus grafting plants. Ks2 and Dl2 represent 'Kanshu' and Diospyros lotus, respectively, in 'Kanshu'/Diospyros lotus grafting plants. Error bars: the standard error of three biological replicates. Different letters: significantly different at $P<0.01$ by Duncan's multiple range tests

proteins) genes exhibited elevated expression in the 'Kanshu' scion tissues from the 'Nantong-xiaofangshi' graft combination. In contrast to the RNA-seq analysis, the spindly gene, which enhances the inhibitory effect of DELLA and negatively regulates gibberellic acid-mediated signaling, was highly upregulated in the KS1 samples relative to the KS2 samples (Fig. 4a). Regarding auxin signal transduction, the expression of auxin-responsive protein IAA (AUX/IAA) and auxin-responsive GH3 gene family members (TR76662|c4_g2_i1; TR65617|c0_g1_i1, TR25117|c0_g1_i2) was elevated in the scion samples from the 'Nantong-xiaofangshi' graft combination. Auxin response factor $(A R F)$ genes were upregulated (TR45847| c2_g3_i1) in the scion and downregulated (TR76050| c4_g1_i2) in the interstock. The expression of the SAUR family protein (SAUR) gene (TR65681|c1_g1_i1) was higher in KS1 than in KS2. Additionally, the transcript levels of two IAA-amino acid hydrolase genes (TR30838| c0_g9_i1, TR44811|c0_g1_i2) were higher in KS1 than in KS2 (Fig. 4b).

\section{Genetic transformation and functional analysis of DkGA2ox1 in tobacco}

DkGA2ox1 (TR54490|c1_g1_i1) expression was elevated in scions ('Kanshu') grafted on the 'Nantong-xiaofangshi' interstock. The functional role of the expression, however, is unclear. Phylogenetic analysis was used to study the biological function of $D k G A 2 o x 1$. Our phylogenetic analysis suggests that TR54490|c1_g1_i1 (DkGA2ox1) is homologous to Camellia lipoensis GA2ox2 (AHZ13201.1) and Camellia sinensis GA2ox2 (ASJ80914.1) and belongs to the same subfamily as Arabidopsis thaliana GA2ox1 (NP177965.1), GA2ox2 (NP174296.1) and GA2ox3 (NP181002.1) (Fig. 5). GA2ox is the principle enzyme involved in the inactivation of bioactive GAs, and GA plays a critical role in stem growth and elongation. Therefore, a functional analysis of this gene was conducted in transgenic tobacco. The results indicated that $D k G A 2 o x 1$ was successfully integrated into the tobacco genome and was expressed in the transgenic lines of tobacco (Fig. S6). Two independent transgenic tobacco lines were selected for further study.

Compared with the growth of the wild type, the growth of the transgenic lines was slow over 60-90 days after transplanting (Fig. 6a). In addition, the transgenic lines exhibited significant dwarfism, compact leaf types, and delayed flowering after 90 days of growth in the greenhouse (Fig. 6b). The phenotypic analysis demonstrated that the 1-1 and 1-3 transgenic lines of tobacco were $38.90 \%$ and $59.03 \%$ shorter than the wild-type plants, respectively. In addition, the internodal length of the transgenic lines was $13.15-60.30 \%$ shorter than the internode length of the wild-type plants. In contrast, the stem diameter in the transgenic lines was $11.92-57.42 \%$ greater than that in the wild-type plants. Finally, leaf length was $23.27-47.07 \%$ shorter in the transgenic lines, while leaf width increased by $21.79-25.00 \%$ (Table 3 ). 


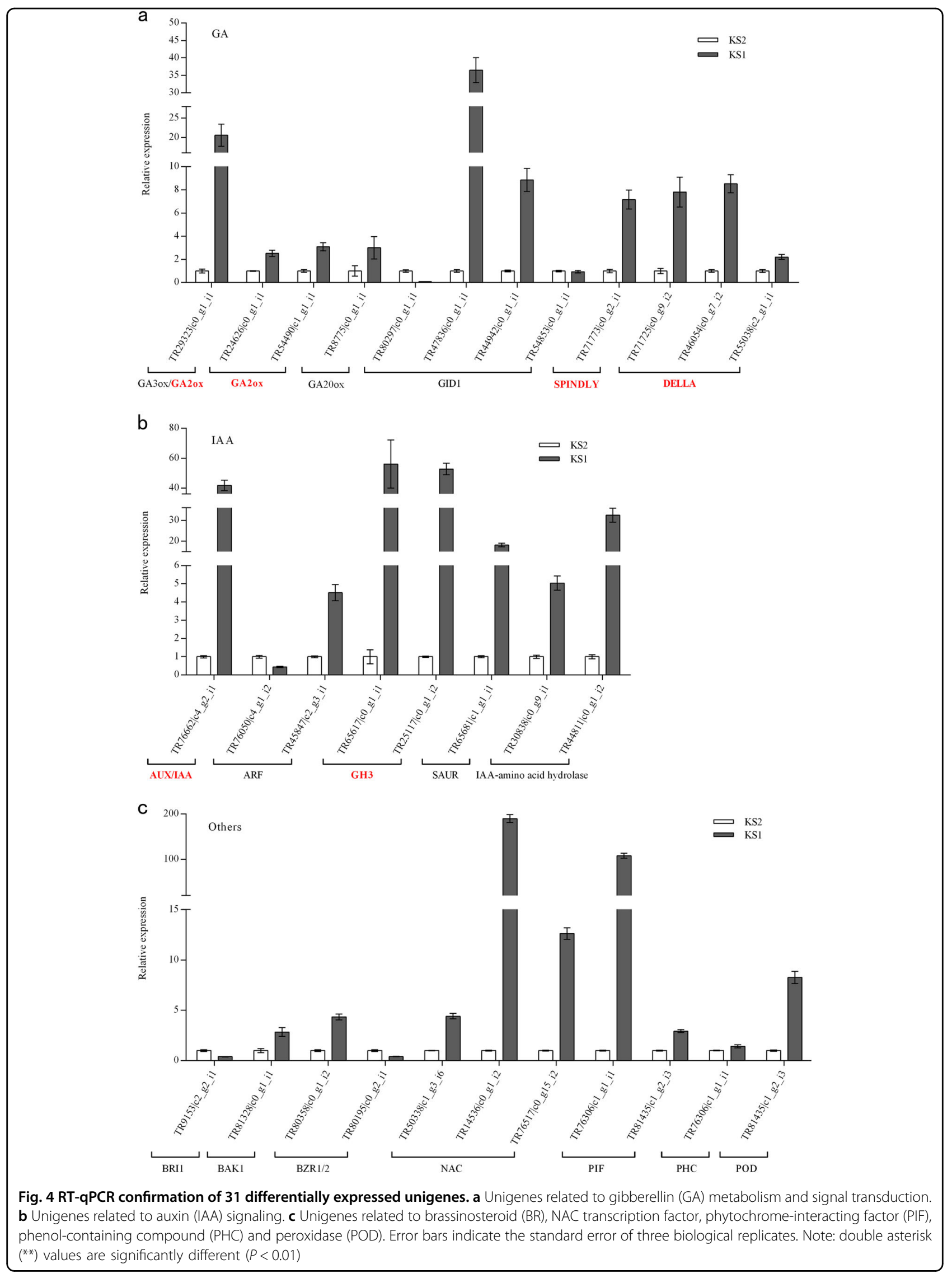




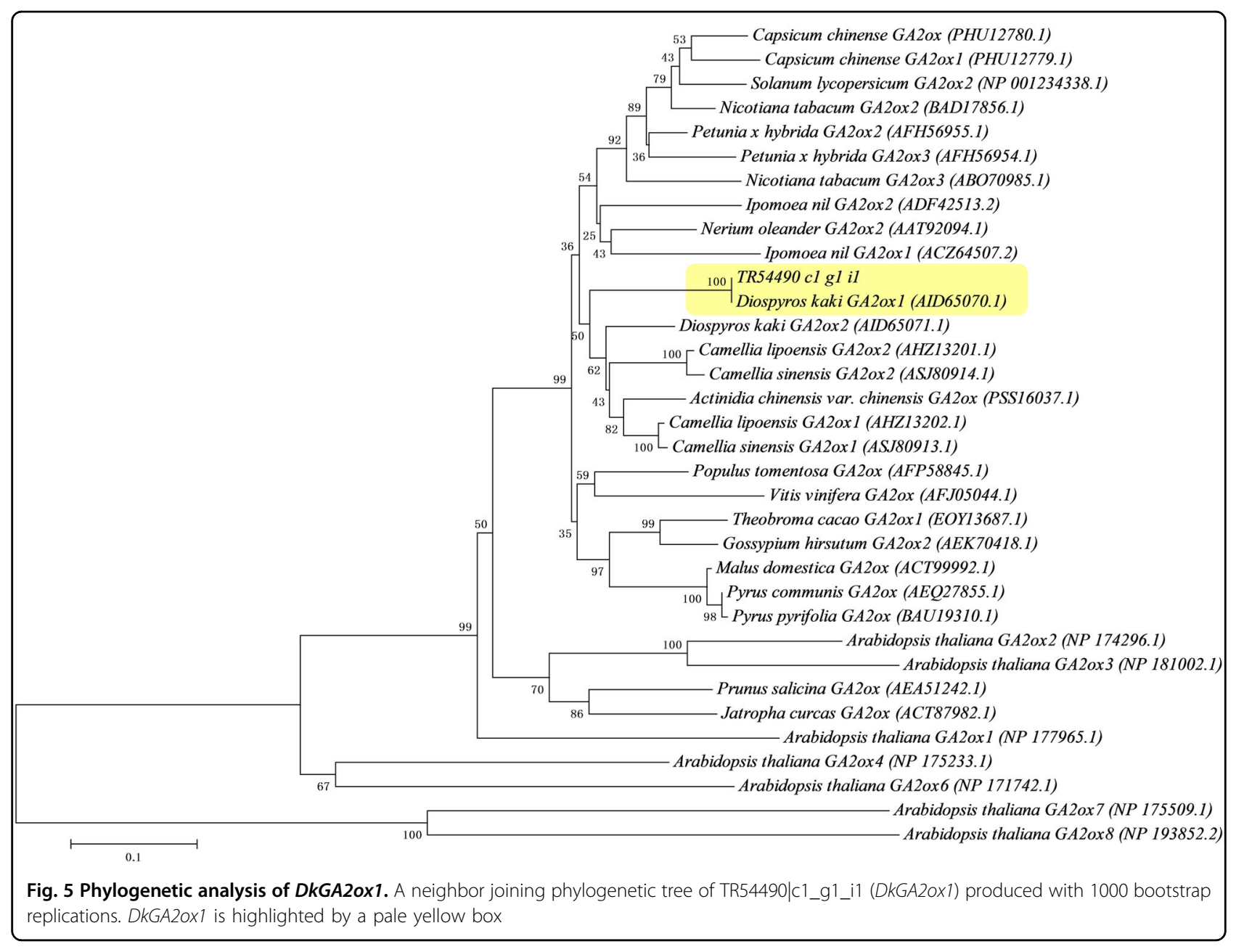

The transgenic and wild-type tobacco plants received two applications of $100 \mathrm{mg} \cdot \mathrm{l}^{-1} \mathrm{GA}_{3}$ each week starting at 30 days after transplantation to the greenhouse. Thirty days after the first application of exogenous $\mathrm{GA}_{3}$, the internode length in the transgenic plants began to increase rapidly, and treated plants flowered normally (Fig. 6c, d). Thus, it appeared that the $\mathrm{GA}_{3}$ application restored the normal phenotype to the transgenic plants.

GA levels were significantly lower in the transgenic lines than in wild-type plants. Overall, the transgenic GA levels were $65-70 \%$ of the levels observed in wild-type plants (Fig. 6e). We suggest that the overexpression of $D k G A 2 o x 1$ in tobacco effectively inhibited the synthesis of GA in the plant, which resulted in the production of shorter plants. Additionally, we analyzed the gene expression of several key enzymes that are involved in GA biosynthesis. The heterologous overexpression of $D k G A 20 x 1$ enhanced the expression of the native $N t G A 20 o x$ and NtGA3ox genes in transgenic tobacco plants compared to wild-type plants, and the increased expression of $N t G A 3 o x$ was more evident than that of NtGA20ox (Fig. 6f).

\section{Cleaved amplified polymorphic sequence analysis of the GA2ox gene}

GA2ox1 genes were amplified from Diospyros lotus, 'Nantong-xiaofangshi', and 'Kanshu' tissue samples based on the sequence of DkGA2ox1. The polypeptides encoded by these genes comprise 332 amino acid residues, including regions composed of PLN02156 (gibberellin 2beta-dioxygenase), Pcbc, (Isopenicillin $\mathrm{N}$ synthase and related dioxygenases), DIOX_N (non-haem dioxygenase in morphine synthesis $\mathrm{N}$-terminal), and 2OG-FeII_Oxy (2OG-Fe(II) oxygenase superfamily). The genes shared 99.26\% sequence identity and contained 25 single nucleotide polymorphisms (Fig. S7). As minor differences were difficult to distinguish by RT-qPCR, restriction enzyme sites within the sequences were identified, and the ApaI cut site was used to distinguish among DkGA2oxI ('Nantong-xiaofangshi'), DLGA2ox1 (Diospyros lotus), and 


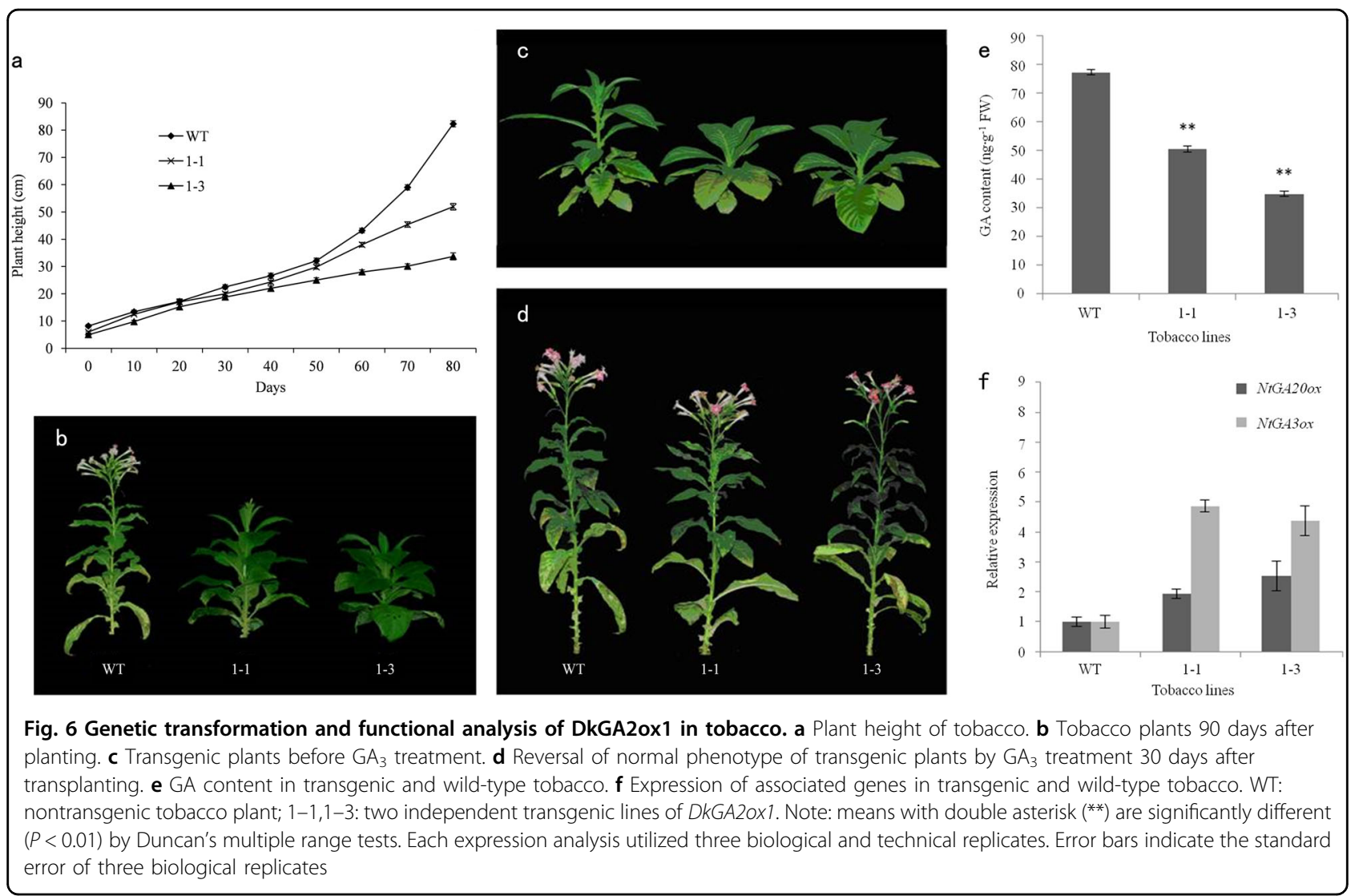

Table 3 Phenotype characteristics of DkGA2ox1 transgenic tobacco

\begin{tabular}{|c|c|c|c|}
\hline & \multirow[t]{2}{*}{ WT } & \multicolumn{2}{|l|}{$D k G A 20 \times 1$} \\
\hline & & $1-1$ & $1-3$ \\
\hline Plant height (cm) & $82.33 \pm 2.59$ & $50.3 \pm 2.69^{* *}$ & $33.73 \pm 1.33^{* *}$ \\
\hline Internodal length $(\mathrm{cm})$ & $4.03 \pm 0.12$ & $3.5 \pm 0.1 * *$ & $1.6 \pm 0.1^{* *}$ \\
\hline Stem diameter (mm) & $9.23 \pm 0.15$ & $10.33 \pm 0.15^{* *}$ & $14.53 \pm 0.47^{* *}$ \\
\hline Leaf length (cm) & $24.37 \pm 1.59$ & $18.70 \pm 0.55^{* *}$ & $12.9 \pm 0.66^{* *}$ \\
\hline Leaf width (cm) & $8.4 \pm 0.26$ & $10.5 \pm 0.1^{* *}$ & $10.23 \pm 0.15^{* *}$ \\
\hline Leaf length/width & $2.9 \pm 0.10$ & $1.78 \pm 0.04^{* *}$ & $1.26 \pm 0.03^{* *}$ \\
\hline
\end{tabular}

Note: means with double asterisk $(* *)$ are significantly different $(P<0.01)$ WT: nontransgenic tobacco plant; 1-1,1-3: transgenic tobacco plants of DkGA2ox1

KSGA2ox1 ('Kanshu'). The enzyme digest fragments are shown in Fig. 7a.

Since the fragment used to distinguish the genes was only $24 \mathrm{bp}$, a cleaved amplified polymorphism sequence (CAPS) marker was designed to distinguish DkGA2ox1 from the other two sequences. The CAPS marker allowed us to demonstrate that the DkGA2ox1 transcript was transported from the interstock ('Nantong-xiaofangshi') to the scion ('Kanshu'). The $219 \mathrm{bp}$ fragment amplified from N.x cDNA was cleaved into three fragments of 24, 68 , and $127 \mathrm{bp}$, while the fragment amplified from 'Kanshu' and Diospyros lotus cDNA was cleaved into two fragments of 92 and 127 bp (Fig. 7a). RT-PCR restriction fragment length polymorphism (RFLP) analysis of 'Nantong-xiaofangshi' revealed a unique restriction band pattern when subjected to polyacrylamide gel electrophoresis (Fig. 7b lanes 2-4). The $92 \mathrm{bp}$ fragment in 'Nantongxiaofangshi' may occur due to incomplete enzyme digestion or may contain both mRNAs of GA2ox. Relative to trees without an interstock (KS2) (Fig. 7b lane 4), CAPS analysis exhibited $24,68,92,127$, and 219 bp fragments in the scion of the 'Kanshu'/N.x/Diospyros lotus graft combination (KS1) (Fig. 7b lane 5). This indicated that the scion had incorporated fragments originating from both the scion and the interstock plants. DkGA2ox1 transcripts produced in the interstock can be transported from the $\mathrm{N}$. $\mathrm{x}$ interstock to the 'Kanshu' scion.

To further examine the transmission of DkG2ox1 transcripts within grafted plants, samples from a twice grafted, $82 \mathrm{~cm}$ tall 'Kanshu'/N.x/Diospyros lotus tree were collected from the scion every $10 \mathrm{~cm}$. In addition, samples were collected from the two graft unions and interstocks (Fig. 7c). DkGA2ox1 transcripts were PCR amplified and purified for CAPS analysis to discriminate SNPs in 


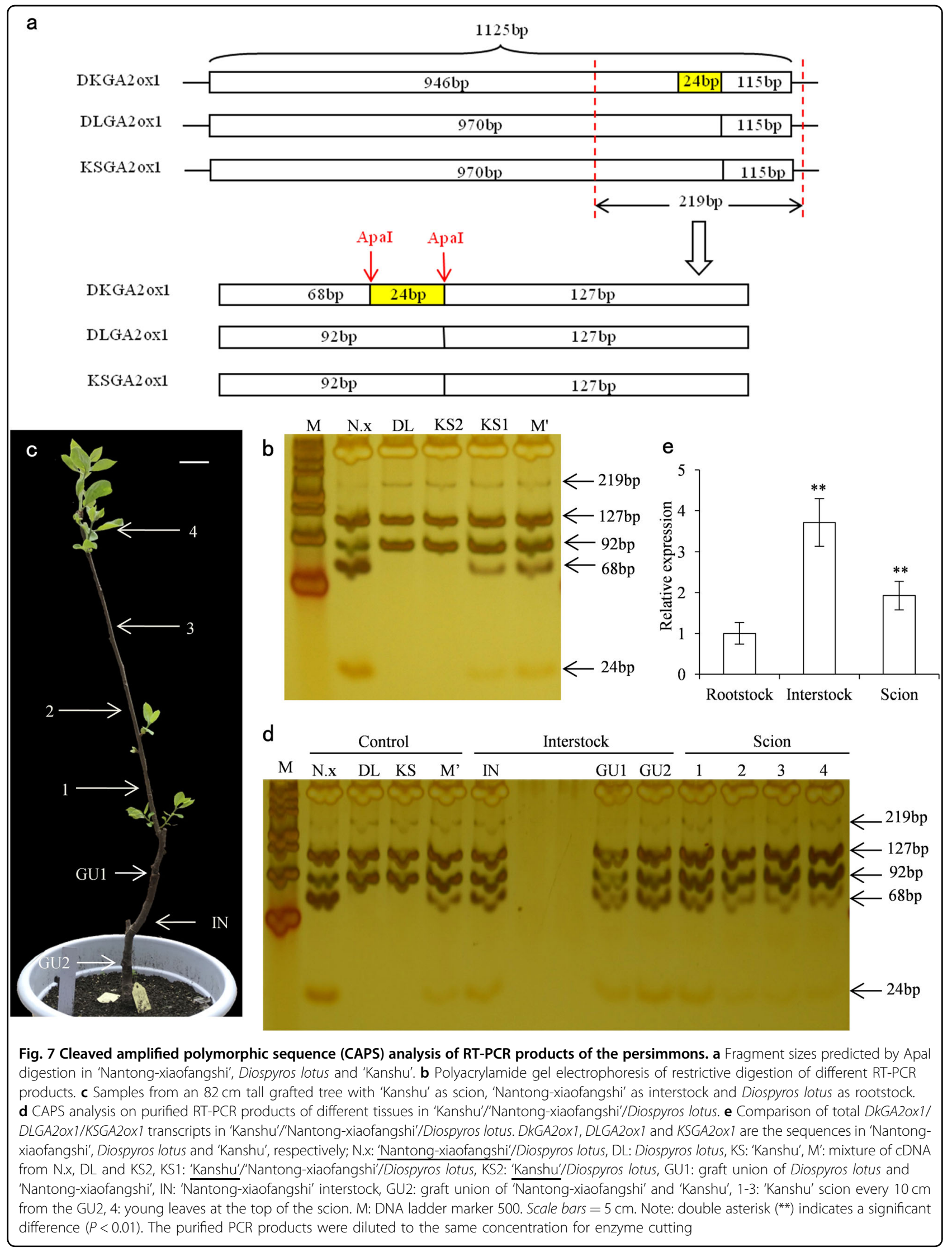


different locations. Fragments of $24 \mathrm{bp}$ and $68 \mathrm{bp}$, which were present in the 'Nantong-xiaofangshi' interstock, were detected in the two graft unions, young leaves at the top of the scion, and scion tissues at different distances from the graft union. These results indicate that endogenous DkGA2ox 1 mRNA originating in the interstock can be transported from the interstock to $10-40 \mathrm{~cm}$ across the graft union into the scion tissues (Fig. 7d). Moreover, in the graft combination 'Kanshu'/'Nantong-xiaofangshi'/ Diospyros lotus, the total expression of DkGA2ox1, DLGA2ox1, and KSGA2ox1 (TR54490|c1_g1_i1) was highest in the interstock tissue samples (Fig. 7e).

\section{Discussion}

The growth and development of plants are regulated in a coordinated manner by phytohormones and environmental factors ${ }^{62}$. Plant hormones such as IAA, GA, ABA, $C T K$, and BR participate in regulating plant growth. Many studies have reported that plant dwarfing is a result of the differential expression or interruption of genes that play a role in phytohormone metabolism ${ }^{63}$ and signal transduction ${ }^{64}$. Despite the use of 'Nantong-xiaofangshi' as an interstock in sweet persimmon dwarfing cultivation, the physiological and molecular change caused by this interstock is poorly understood. Moreover, some studies have shown that xylem vessel characteristics appear to determine the hydraulic conductance capacity and influence vegetative growth ${ }^{7}$. Morphological analysis of the graft junction showed the effective sealing of the 'Kanshu' scion with the interstock and rootstock (Fig. S8). The phloem and most of the xylem were completely sealed at the graft junctions, which had a low effect on transport between the scion and interstock/rootstock. Interestingly, the water conductance in the tree with 'Nantong-xiaofangshi' as an interstock was markedly lower than that in trees without an interstock (Fig. S5), and this decreased conductance could reduce substance transport and influence phytohormone levels in the scion. In addition, the hormone contents in scions grafted to the interstock could reflect the water conductance under less stressful conditions (Fig. 3). This result indicated that the number of graft unions and the interstock variety probably also affected the water conductance of the grafting area to a certain extent, and the low water conductance in the grafts with interstocks could be a major reason for dwarfing in response to 'Nantong-xiaofangshi' interstock grafting.

The effect of grafting on plants was evident throughout the entire year, and over the course of multiple seasons, the plant populations were stable. The seasonal change in the growth speed of plants differed, and the most obvious change was observed during the rapid elongation of shoots. At the end of April and during early May, the scion growth showed obvious differences between the two grafted combinations. This period is one of the stages in which the interstock has a significant effect on growth. In the present study, GO and KEGG analyses of unigenes revealed that the unigenes were involved in pathways including plant hormone signal transduction and metabolism. Unigenes in the pathways of diterpenoid biosynthesis, tryptophan metabolism, and GA, IAA hormone signal transduction are probably the candidate genes causing the dwarfing effect of interstocks on tree growth. Plant hormones and the expression of hormone-related genes significantly changed in scion tissues when 'Nantong-xiaofangshi' was used as an interstock (Figs. 3, 4). This result indicates that the "Nantong-xiaofangshi" interstock plays an important role in modifying the balance of phytohormones in grafted plants and also indicates that the physical distance to the shoot meristem might affect transcript and hormone levels.

Even if the physical distance to the shoot meristem has an impact on plants, the IAA and GA levels in KS1 (grafted with an interstock) were still lower than those in KS2 (grafted without an interstock). Therefore, this point may not be the main reason for the change in hormones in the tissue for research. In the present study, we primarily focused on the role of IAA and GA, as well as their related genes, in the dwarfing effect of the 'Nantongxiaofangshi' interstock.

IAA has a profound influence on the regulation of scion growth by an interstock or rootstock ${ }^{2,13}$. In this study, IAA levels in the scion and rootstock were lower in plants where 'Nantong-xiaofangshi' was used as an interstock than in those with no interstock (Fig. 3a). Michalczuk ${ }^{64}$ also detected a low IAA level in scions after they were grafted on a dwarf rootstock ${ }^{65}$. Early isotope labeling experiments demonstrated that dwarfing rootstocks block IAA transport to roots and that the content of IAA received by the roots of dwarfing rootstocks is lower than that in roots of vigorous rootstocks ${ }^{20}$. The polar transport of IAA can be impeded by dwarfing interstocks and result in the production of a dwarf phenotype ${ }^{13}$. The dwarfing mechanism of interstocks vs. rootstocks may be different, but both can reduce the level of IAA present in scions ${ }^{66}$. We hypothesize that the 'Nantong-xiaofangshi' interstock influenced the distribution of IAA and its transport to the rootstock, thereby resulting in the dwarfing of the scion.

Auxin can induce the expression of several auxinresponsive genes that fall into three different categories: Aux/IAA, GH3, and SAUR gene families, as well as ARF genes, the principle target of auxin ${ }^{67}$. The expression of $A U X / I A A$ and $G H 3$ genes was increasingly downregulated with increasing IAA levels and vigorous growth (Fig. 4b). This downregulation was due to the enhanced expression of $A U X / I A A$ genes, which act as repressors of auxin transduction and GH3 proteins, deactivating IAA and impeding auxin signaling by catalyzing the disassociation 
of IAA $^{68}$. Liu et al. ${ }^{12}$ investigated gene expression in 'Shatangju' mandarin grafted on different rootstocks and demonstrated that the expression of $G H 3$ genes and the AUX/IAA family gene, IAA4, was downregulated as growth and vigor increased ${ }^{8}$. Based on the abovementioned studies and the role of auxin in the regulation of plant growth, the altered expression of $A U X / I A A$ and GH3 family genes probably plays a crucial role in dwarfing. Notably, several $A R F$ genes were upregulated and downregulated in our study. One possible interpretation of the contrasting expression profiles in response to the same signal is that $A R F$ genes may play diverse functions in plants. Five of the ARFs (ARF5-8 and 19) in Arabidopsis function as transcriptional activators, while the remaining $18 A R F \mathrm{~s}$ act as repressors ${ }^{69}$. ARF transcription factors may modulate $G H 3$ genes, whose expression is correlated with plant growth and development ${ }^{70}$. This premise is in agreement with the results of our qPCR analysis (Fig. 4b). Upregulated expression of $A R F$ genes may stimulate $G H 3$ transcription in plants using 'Nantong-xiaofangshi' as an interstock, thereby hindering the transmission of auxin signaling and leading to dwarfing of the scion. The expression patterns of $A R F$ genes need further investigation.

GA promotes stem elongation, and its effect is closely related to IAA, i.e., IAA acts with GA in a synergistic manner, and GA affects the activity of $\mathrm{IAA}^{71}$. In the current study, GA levels in the scion and interstock tissues of 'Kanshu'/'Nantong-xiaofangshi'/Diospyros lotus grafted plants were lower than those in the scion tissues of 'Kanshu'/Diospyros lotus grafted plants. A negative correlation was observed between the GA levels and scion dwarfing (Fig. 3b). GAs can be transported over long distances in grafted plants and can regulate growth ${ }^{22,23}$. As previously mentioned, dwarfing rootstocks and interstocks can decrease the basipetal transport of IAA to the roots. Hooijdonk et al. ${ }^{23,24}$ confirmed this premise and further indicated that GA synthesis and transmission are also suppressed by rootstocks, resulting in decreased levels of GA in the scion and in dwarfed plants. We found a positive correlation between the levels of GA and IAA in the scion tissues of the two graft combinations. Auxin promotes the synthesis and transduction of GA, which is necessary for the biosynthesis of active forms of $\mathrm{GA}^{71}$. Exogenous application of an auxin transport inhibitor has been shown to remarkably decrease the GA levels in shoots $^{72}$. Therefore, we hypothesize that GA synthesis and transport to aboveground plant parts (scions) are limited by the 'Nantong-xiaofangshi' interstock, which thus reduces GA levels in the scion and results in a dwarf phenotype.

In addition to hormone analysis, we examined gene expression in different tissues to further understand the dwarfing mechanism. GA2ox can catalyze the conversion of bioactive GA and its precursors into irreversible forms of inactive $\mathrm{GA}^{73}$. Liu et al. ${ }^{8}$ reported that the expression of GA2ox1 was negatively correlated with plant growth and vigor. GA2ox may act as a substitute for GID1 in the deactivation of bioactive gibberellins with a high affinity ${ }^{47}$. In our study, the relatively low levels of GA in the scion of 'Kanshu'/'Nantong-xiaofangshi'/Diospyros lotus grafted plants were probably caused by the upregulation of GA2ox in the scion tissues, which may have led to the inactivation of bioactive GA (Fig. 3b). GA20ox and GA3ox regulate the last stage of GA biosynthesis and thus control the level of bioactive $\mathrm{GA}^{74}$. Interestingly, the expression of both GA20ox and GA3ox increased in the scions of plants where 'Nantong-xiaofangshi' was used as an interstock (Fig. 4a). Ou et al. ${ }^{74}$ also reported that GA3ox expression levels were not downregulated in dwarf varieties ${ }^{75}$. These results indicate that GA20ox and GA3ox expression are not the only factors impacting the level of GA. The flux of active GA is due to the balance between the rate of bioactive GA synthesis and deactivation. Therefore, if the GA inactivation rate is faster than the GA synthesis rate, low levels of GA are likely to be observed in the scion of 'Kanshu'/'Nantong-xiaofangshi'/Diospyros lotus grafted plants.

Notable GA signal transduction-related unigenes were those encoding DELLA proteins, which act as central repressors of GA signaling ${ }^{74}$. GA signaling can be moderated by the gibberellic acid insensitive (GAI) repressor, which possesses the highly conserved DELLA domain. Atgai overexpressing Arabidopsis plants exhibit a dwarf phenotype ${ }^{42}$. After extensive research, $\mathrm{Li}$ and colleagues demonstrated that GAI mRNA can be transported in phloem tissues across a graft union and induce a dwarf phenotype in grafted scions ${ }^{36,37,42}$. Therefore, we speculate that a 'Kanshu' scion grafted on a 'Nantong-xiaofangshi' interstock accumulates more DELLA transcripts that without an interstock, and this inhibits the transmission of GA signals. This process may be partially responsible for the dwarfing of the scion in persimmon when 'Nantong-xiaofangshi' is used as an interstock. Our analysis of gene expression also indicated that the expression of a gene encoding SPINDLY (SPY) was similar to the expression of DELLA genes (Fig. 4a). SPINDLY $(S P Y)$ also plays an important role in repressing GAinduced stem elongation ${ }^{76}$. The negative regulation of the GA response pathway by the $S P Y$ gene is accomplished by the activation of the suppressive function of DELLA proteins $^{76}$. Therefore, the expression of SPY may activate DELLA proteins, thereby affecting GA signaling and inducing the dwarf phenotype.

GA2ox is currently considered to promote the deactivation of bioactive GAs, or their precursors, to inactive forms. GA2ox genes have been studied in several species, including Arabidopsis ${ }^{77}$, rice $^{78}$, Populus ${ }^{79}$, and plum ${ }^{47}$, in 
which GA2ox expression strongly impeded the biosynthesis of bioactive GA and was associated with a dwarf phenotype. Previous research has identified several mechanisms for GA inactivation, the most prevalent of which is $2 \beta$-hydroxylation. GA2oxs, which are responsible for this activity, are divided into two groups: one acts on C19-GAs, and the other acts on C20-GAs ${ }^{21}$. GA2oxs acting on C19-GAs to form inactive products can be further divided into two subgroups according to substrate $^{80}$. The first subgroup deactivates $3 \beta$-hydroxy bioactive compounds $\left(\mathrm{GA}_{1}\right.$ and $\left.\mathrm{GA}_{4}\right)$, including AtGA2ox1-3, and the other group acts on C19-GA immediate non3 3 -hydroxylated precursors $\left(\mathrm{GA}_{9}\right.$ and $\mathrm{GA}_{20}$ ), including $A t G A 2 o x 4,6^{77}$. The GA2oxs acting on C20-GAs $\left(\mathrm{GA}_{12}\right.$ and $\left.\mathrm{GA}_{53}\right)$ are considered as a separate family from C19-GA2oxs and include AtGA2ox $7-8^{77}$. Based on sequence phylogeny, DkGA2ox1 belongs to the first subgroup, with C19-GAs as substrates (Fig. 5).

In the present study, GUS detection and RT-PCR analyses indicated that the exogenous $D k G A 2 o x 1$ gene was transferred into tobacco successfully and was not present in the wild-type plant. The expression of DkGA2ox1 in transgenic tobacco resulted in a dwarf phenotype and other dwarfing traits that could be overcome by the exogenous application of $\mathrm{GA}_{3}$ (Fig. 6a-e). These results confirmed that the dwarf phenotype was caused by the overexpression of DkGA2ox1, which reduced bioactive GA levels. The overexpression of $A t G A 2 o x 7$ and AtGA2ox8 in Arabidopsis and OsGA2ox6 and OsGA2ox5$O X$ in rice resulted in similar dwarfing characteristics ${ }^{77,78}$. Additionally, expression analysis of GA-synthesis genes indicated that GA20ox and GA3ox are upregulated in GAdeficient and GA-insensitive mutants ${ }^{81,82}$. In our study of transgenic tobacco, the expression of native $N t G A 20 o x$ and $N t G A 3 o x$ genes was upregulated in transgenic lines overexpressing DkGA2ox1 (Fig. 6f). This was probably due to a feedback regulation mechanism induced by the decrease in the level of endogenous GA.

Some mobile macromolecules, such as RNAs and proteins, have been shown to be transported in the vascular system and pass through a graft union to their targets in the grafted scion $^{31,83,84}$. To further explore the role of GA2ox in the dwarfing mechanism of the 'Nantongxiaofangshi' interstock, we determined whether GA2ox mRNA was transported from the interstock to the scion, as that may be one of the mechanisms responsible for the dwarfing of the scion. Studies have shown that many different mRNAs can be transported across the graft union in adult plants ${ }^{5,34,85}$. Therefore, we used adult grafted persimmon plants as our experimental material. The results indicated that native $D k G A 2 o x 1$ mRNA was transported from the 'Nantong-xiaofangshi' interstock to the 'Kanshu' scion (Fig. 7). El-Sharkawy et al. ${ }^{47}$ grafted 'Early Golden' (EG) on 'DGO24', a dwarf plum hybrid rootstock, and observed high levels of PslGA2ox expression, which enhanced the accumulation of PslGA2ox in the scion and resulted in a compact stature phenotype in the scions of grafted trees. They suggested that the 'DGO24' rootstock may be involved in the inactivation of bioactive GAs within the scion, a premise that was supported by the low amount of active GAs in EG/D trees relative to control trees ${ }^{47}$. Increased expression of GA2ox1 was observed in the scion grafted onto the 'Nantong-xiaofangshi' interstock (Fig. 4a). This indicates that the 'Nantong-xiaofangshi' interstock can enhance the accumulation GA2oxl in the scion, and the high expression of GA2ox1 in KS1 may be affected by the transport of DkGA2ox1 transcripts.

In the 'Kanshu'/'Nantong-xiaofangshi'/Diospyros lotus grafted plants, the content of GA in the interstock was much lower than that in the scion tissues. This may be due to the significantly higher expression of GA2ox1 in the interstock than in the scion tissues (Figs. 3b, 7e). In addition, the GA level in the rootstock with interstock was higher than that in the rootstock without interstock (Fig. 4a). These observations suggest that GA transported from the rootstock may be deactivated in the interstock, thus affecting the bioactive GA content in the scion. GA levels in the scion were higher than those in the interstock, but this may be due to the nondirectional transport of GA, with equal movement of GA in both an acropetal and a basipetal direction ${ }^{86}$. Furthermore, DkGA2ox1 mRNA produced in the interstock can be transported to scion stems and leaves at least $10-30 \mathrm{~cm}$ away from the graft union in twice-grafted trees (Fig. 7c, d). This suggests that the transport of $D k G A 2 o x 1$ mRNA may be similar to the transport of GAI and NAC mRNA, which was also shown to be transported long distances from the source to sink tissues where they were accumulated ${ }^{36,64,87}$. The transport of mRNA in vascular tissues is a complex biological process. Although there are differences between herbaceous and woody plants, future experiments using the generated DkGA2ox1-overexpressing transgenic tobacco plants and wild-type tobacco plants to test whether grafting causes dwarfing of wild-type tobacco scion can further clarify this process.

In conclusion, the 'Nantong-xiaofangshi' interstock governed the metabolism, transport, and distribution of endogenous hormones, resulting in the reduced growth (dwarfing) of the grafted scion. In grafted plants, water conductance mostly impacted dwarfing by the interstock because of substance transport, including the transport of phytohormones to the scion, primarily depends on the xylem stream. Additionally, the phytohormone signaling level in the scion could reflect the dwarfing phenotype, which is possibly affected by water conductance. The dwarfing interstock altered gene expression associated with the metabolism and signal transduction of IAA and 
GA and influenced differential phytohormone levels in the two different graft combinations. Regarding the differential expression of the hormone-related genes, we observed that the GA2ox gene was highly expressed in the scion. One of the GA2ox genes was annotated as DkGA2ox1 in Diospyros kaki, and the overexpression of this gene in transgenic plants resulted in a dwarf phenotype. Furthermore, DkGA2ox1 transcripts originating in the interstock plant part were identified in the scion, thus indicating that the transport of DkGA2ox1 transcripts could have an additional effect on dwarfing. However, the contribution of mobile transcripts is still unknown. These pathways may cooperatively cause the dwarfing phenomenon exhibited when a dwarfing interstock is utilized. Our studies lay the foundation for additional studies on the dwarfing mechanism of the 'Nantong-xiaofangshi' interstock, in which the details of the proposed mechanisms of dwarfing are fully elucidated and demonstrated.

\section{Acknowledgements}

This study was supported by the Science and Technology Special Project in North Jiangsu Research Funds (SZ-LYG2017004) and the National Public Welfare Industry (Agriculture) Project Special Scientific Research Funds (201203047). We thank Journal Doctors Inc. (www.journaldoctors.com) for proofreading and editing this manuscript.

\section{Author contributions}

S.Q. and Y.S. designed the project. Y.S. performed most of the experiments. X.T. performed the transgenic experiments. Y.S., X.T., X.L., and F.L. prepared the materials. Y.S. and S.Q. wrote the manuscript. W.Z., A.X., Z.G., and X.Y. revised the manuscript.

\section{Author details}

'College of Horticulture, Nanjing Agricultural University, 210095 Nanjing, Jiangsu, China. ${ }^{2}$ Jiangsu Key Laboratory for Horticultural Crop Genetic Improvement, 210014 Nanjing, China. ${ }^{3}$ Jiangsu Key Laboratory for the Research and Utilization of Plant Resources, Institute of Botany, Jiangsu Province and Chinese Academy of Sciences, 210014 Nanjing, China

\section{Conflict of interest}

The authors declare that they have no conflict of interest.

Supplementary Information accompanies this paper at (https://doi.org/ 10.1038/s41438-019-0133-7).

Received: 12 April 2018 Revised: 9 January 2019 Accepted: 23 January 2019 Published online: 01 May 2019

\section{References}

1. Koshita, Y. et al. Selection of interstocks for dwarfing Japanese persimmon (Diospyros kaki Thunb.) trees. J. Jpn Soc. Hortic. Sci. 76, 288-293 (2007).

2. Koepke, T. \& Dhingra, A. Rootstock scion somatogenetic interactions in perennial composite plants. Plant Cell Rep. 32, 1321-1337 (2013).

3. Tetsumura, T. et al. Growth and production of adult Japanese persimmon (Diospyros kaki) trees grafted onto dwarfing rootstocks. Sci. Hortic.-Amst. 187, 87-92 (2015).

4. Yakushiji, H., Azuma, A., Sugiura, H., Yamasaki, A. \& Koshita, Y. Comparison of promising dwarfing rootstocks for 'Fuyu' Japanese persimmon trees. Acta 1130, 469-472 (2016)

5. Jensen, P. J. et al. Rootstock-regulated gene expression patterns in apple tree scions. Tree Genet Genomes 6, 57-72 (2010).
6. Knabel, M. et al. Genetic control of pear rootstock-induced dwarfing and precocity is linked to a chromosomal region syntenic to the apple Dw1 loci. BMC Plant Biol. 15, 230 (2015).

7. Tombesi, S., Johnson, R. S., Day, K. R. \& Dejong, T. M. Relationships between xylem vessel characteristics, calculated axial hydraulic conductance and sizecontrolling capacity of peach rootstocks. Ann. Bot.-Lond. 105, 327-331 (2010).

8. Liu, X. Y., Li, J., Liu, M. M., Yao, Q. \& Chen, J. Z. Transcriptome profiling to understand the effect of citrus rootstocks on the growth of 'Shatangju' Mandarin. PLOS ONE 12, e0169897 (2017).

9. Lauri, P. E. et al. Genetic determinism of anatomical and hydraulic traits within an apple progeny. Plant Cell Environ. 34, 1276-1290 (2011).

10. Jones, O. Effect of dwarfing interstocks on xylem sap composition in apple trees: effect on nitrogen, potassium, phosphorus, calcium and magnesium content. Ann. Bot.-Lond. 40, 1231-1235 (1976).

11. Lochard, R. \& Schneider, G. Stock and scion growth relationships and the dwarfing mechanism in apple. Hortic. Rev. 3, 315-375 (2011).

12. Liang, F. H. et al. Different rootstocks and physiological and biochemical change of SUM cherry in greenhouse environment. Adv. Mater. Res. 886, 304-308 (2014).

13. Li, H. L. et al. Possible roles of auxin and zeatin for initiating the dwarfing effect of M9 used as apple rootstock or interstock. Acta Physiol. Plant. 34, 235-244 (2012).

14. Soumelidou, K., Battey, N. H., John, P. \& Barnett, J. R. The anatomy of the developing bud union and its relationship to dwarfing in apple. Ann. Bot.Lond. 74, 605-611 (1994).

15. Cohen, S., Naor, A., Bennink, J., Grava, A. \& Tyree, M. Hydraulic resistance components of mature apple trees on rootstocks of different vigours. J. Exp. Bot. 58, 4213-4224 (2007).

16. Chaplin, M. \& Westwood, M. Effects of pyrus species and related genera rootstocks on mineral uptake in 'Bartlett'pear 1. J. Plant. Nutr. 2, 335-346 (1980).

17. Blake, P., Webster, T. \& Atkinson, C. Understanding the way rootstocks dwarf fruit trees. Annu. Report. Hortic. Res Int. 96, 32-35 (1997).

18. Aloni, B., Cohen, R., Karni, L., Aktas, H. \& Edelstein, M. Hormonal signaling in rootstock-scion interactions. Sci. Hortic-Amst. 127, 119-126 (2010).

19. Tworkoski, T. \& Fazio, G. Hormone and growth interactions of scions and sizecontrolling rootstocks of young apple trees. Plant Growth Regul. 78, 105-119 (2016).

20. Kamboj, J. S., Browning, G., Quinlan, J. D., Blake, P. S. \& Baker, D. A. Polar transport of $[3 \mathrm{H}]-I A A$ in apical shoot segments of different apple rootstocks. J. Hortic. Sci. 72, 773-780 (1997).

21. Hedden, P. \& Thomas, StephenG. Gibberellin biosynthesis and its regulation. Biochem. J. 444, 11-25 (2012).

22. Regnault, $\mathrm{T}$. et al. The gibberellin precursor $\mathrm{GA}(12)$ acts as a long-distance growth signal in Arabidopsis. Nat. Plants. 1, 15073 (2015).

23. Van Hooijdonk, B. M., Woolley, D. J., Warrington, I. J. \& Tustin, D. S. Initia alteration of scion architecture by dwarfing apple rootstocks may involve shoot-root-shoot signalling by auxin, gibberellin, and cytokinin. J. Hortic. Sci. Biotech. 85, 59-65 (2010).

24. van Hooijdonk, B., Woolley, D., Warrington, I. \& Tustin, S. Rootstocks modify scion architecture, endogenous hormones, and root growth of newly grafted 'Royal Gala' apple trees. J. Am. Soc. Hortic. Sci. 136, 93-102 (2011).

25. Marcon, J. L. et al. Evaluation of the Productive and Vegetative Aspects of the Cultivar 'Imperial Gala' Apple Tree with EM-9 Interstem in Different Lengths. Viii International Symposium on Temperate Zone Fruits in the Tropics and Subtropics. 872, 375-378 (2010).

26. Yu, C., Zhang H. \& Li, H. L. The content of hormone and auxin transport gene pin 1 of SH40 as the interstock of apple. J. China Agric. University. 17, 80-84 (2012).

27. Koshita, Y., Morinaga, K. \& Tsuchida, Y. The early growth and photosynthetic rate of Japanese persimmons (Diospyros kaki L.) grafted onto different interstocks. Sci. Hortic.-Amst. 109, 138-141 (2006).

28. Prassinos, C., Ko, J. H., Lang, G., lezzoni, A. F. \& Han, K. H. Rootstock-induced dwarfing in cherries is caused by differential cessation of terminal meristem growth and is triggered by rootstock-specific gene regulation. Tree Physiol. 29, 927-936 (2009).

29. Tsutsui, H. \& Notaguchi, M. The use of grafting to study systemic signaling in plants. Plant Cell Physiol. 58, 1291-1301 (2017).

30. Kuhn, C., Franceschi, V. R., Schulz, A., Lemoine, R. \& Frommer, W. B. Macromolecular trafficking indicated by localization and turnover of sucrose transporters in enucleate sieve elements. Science 275, 1298-1300 (1997).

31. Notaguchi, M., Higashiyama, T. \& Suzuki, T. Identification of mRNAs that move over long distances using an RNA-Seq Analysis of Arabidopsis/ 
Nicotiana benthamiana Heterografts. Plant Cell Physiol. 56, 311-321 (2015).

32. Gomez, G., Torres, H. \& Pallas, V. Identification of translocatable RNA-binding phloem proteins from melon, potential components of the long-distance RNA transport system. Plant J. 41, 107-116 (2005).

33. Duan, X. W. et al. PbWoxT1 mRNA from pear (Pyrus betulaefolia) undergoes long-distance transport assisted by a polypyrimidine tract binding protein. New Phytol. 210, 511-524 (2016).

34. Kanehira, A. et al. Apple phloem cells contain some mRNAs transported over long distances. Tree Genet Genomes 6, 635-642 (2010).

35. Ham, B. K. et al. A polypyrimidine tract binding protein, pumpkin RBP50, forms the basis of a phloem-mobile ribonucleoprotein complex. Plant Cell 21, 197-215 (2009).

36. Zhang, W. N. et al. Gibberellic acid-insensitive mRNA transport in Pyrus. Plant. Mol. Biol. Rep. 30, 614-623 (2012).

37. $\mathrm{Xu}, \mathrm{H}$. Y. et al. Gibberellic acid insensitive mRNA transport in both directions between stock and scion in Malus. Tree Genet. Genomes 6, 1013-1019 (2010).

38. Duan, X. W. et al. KNOTTED1 mRNA undergoes long-distance transport and interacts with movement protein binding protein $2 \mathrm{C}$ in pear (Pyrus betulaefolia). Plant Cell Tiss. Org. 121, 109-119 (2015).

39. Zhang, W. N., Duan, X. W., Ma, C., Harada, T. \& Li, T. Z. Transport of mRNA molecules coding NAC domain protein in grafted pear and transgenic tobacco. Biol. Plant. 57, 224-230 (2012).

40. Banerjee, A. K. Lin, T. \& Hannapel, D. J. Untranslated regions of a mobile transcript mediate RNA metabolism. Plant Physiol. 151, 1831-1843 (2009).

41. Lucas, W. J., Yoo, B. C. \& Kragler, F. RNA as a long-distance information macromolecule in plants. Nat. Rev. Mol. Cell Bio. 2, 849-857 (2001).

42. Xu, H. Y., Iwashiro, R., Li, T. Z. \& Harada, T. Long-distance transport of gibberellic acid insensitive mRNA in Nicotiana benthamiana. BMC Plant Biol. 13, 165 (2013).

43. Kehr, J. \& Buhtz, A. Long distance transport and movement of RNA through the phloem. Comp. Biochem Phys. A. 146, S240-S240 (2007).

44. Tu, X. et al. A Dwarf Persimmon Cultivar, 'Nantong-xiaofangshi'. V. Int. Symp Persimmon. 996, 139-144 (2013).

45. Dai, W., Sheng, B. \& Zhang, Z. Endogenous hormone contents in standard and dwarf Persimmon (Diospyros kaki Linn. Cv.). Hortscience 31, 684-684 (1996).

46. Dayan, J., Schwarzkopf, M., Avni, A. \& Aloni, R. Enhancing plant growth and fiber production by silencing GA 2-oxidase. Plant. Biotechnol. J. 8, 425-435 (2010).

47. El-Sharkawy, I. et al. Identification and genetic characterization of a gibberellin 2-oxidase gene that controls tree stature and reproductive growth in plum. J. Exp. Bot. 63, 1225-1239 (2012)

48. Grabherr, M. G. et al. Trinity: reconstructing a full-length transcriptome without a genome from RNA-Seq data. Nat. Biotechnol. 29, 644-652 (2011).

49. Pertea, G. et al. TIGR Gene Indices clustering tools (TGICL): a software system for fast clustering of large EST datasets. Bioinformatics 19, 651-652 (2003).

50. Altschul, S. F., Gish, W., Miller, W., Myers, E. W. \& Lipman, D. J. Basic local alignment search tool. J. Mol. Biol. 215, 403-410 (1990).

51. Conesa, A. et al. Blast2GO: a universal tool for annotation, visualization and analysis in functional genomics research. Bioinformatics 21, 3674-3676 (2005).

52. Ye, J. et al. WEGO: a web tool for plotting GO annotations. Nucleic Acids Res. 34, W293-W297 (2006).

53. Mortazavi, A., Williams, B. A., Mccue, K, Schaeffer, L. \& Wold, B. Mapping and quantifying mammalian transcriptomes by RNA-Seq. Nat. Methods 5, 621-628 (2008).

54. You, C. C. et al. Effect of removing superior spikelets on grain filling of inferior spikelets in rice. Front. Plant Sci. 7, 1161 (2016)

55. Tyree, M. T., Patiño, S., Bennink, J. \& Alexander, J. Dynamic measurements of root hydraulic conductance using a high-pressure flowmeter in the laboratory and field. J. Exp. Bot. 46, 83-94 (1995).

56. Gascó, A. et al. Hydraulic kinetics of the graft union in different Olea europaea L. scion/rootstock combinations. Environ. Exp. Bot. 60, 245-250 (2007).

57. Bustin, S. A. et al. The MIQE guidelines: minimum information for publication of quantitative real-time PCR experiments. Clin. Chem. 55, 611-622 (2009).

58. Wang, P. et al. Selection of suitable reference genes for RT-qPCR normalization under abiotic stresses and hormone stimulation in Persimmon (Diospyros kaki Thunb). PLOS ONE 11, e0160885 (2016).

59. Livak, K. J. \& Schmittgen, T. D. Analysis of relative gene expression data using real-time quantitative $P C R$ and the $2-\triangle \Delta C T$ method. Methods 25, 402-408 (2001).

60. Burow, M. D., Chlan, C. A., Sen, P., Lisca, A. \& Murai, N. High-frequency generation of transgenic tobacco plants after modified leaf disk cocultivation withAgrobacterium tumefaciens. Plant Mol. Biol. Rep. 8, 124-139 (1990).
61. Tamura, K., Stecher, G., Peterson, D., Filipski, A. \& Kumar, S. MEGA6: molecular evolutionary genetics analysis version 6.0. Mol. Biol. Evol. 30, 2725-2729 (2013).

62. Sultan, S. E. Phenotypic plasticity for plant development, function and life history. Trends Plant Sci. 5, 537-542 (2000).

63. Dijkstra, C. et al. Over-expression of a gibberellin 2-oxidase gene from Phaseolus coccineus $L$. enhances gibberellin inactivation and induces dwarfism in Solanum species. Plant Cell Rep. 27, 463-470 (2008).

64. Haywood, V., Yu, T. S., Huang, N. C. \& Lucas, W. J. Phloem long-distance trafficking of Gibberellic acid-insensitive RNA regulates leaf development. Plant 1. 42, 49-68 (2005)

65. Michalczuk, L. Indole-3-acetic acid level in wood, bark and cambial sap of apple rootstocks differing in growth vigour. Acta Physiol. Plant. 24, 131-136 (2002).

66. Zhang, H., An, H. S., Wang, Y., Zhang, X. Z. \& Han, Z. H. Low expression of PIN gene family members is involved in triggering the dwarfing effect in $M 9$ interstem but not in M9 rootstock apple trees. Acta Physiol. Plant. 37, 104 (2015).

67. Guilfoyle, T. Plant biology-sticking with auxin. Nature 446, 621-622 (2007).

68. Staswick, P. E. et al. Characterization of an Arabidopsis enzyme family that conjugates amino acids to indole-3-acetic acid. Plant Cell 17, 616-627 (2005).

69. Guilfoyle, T. J. \& Hagen, G. Auxin response factors. Curr. Opin. Plant Biol. 10 453-460 (2007).

70. Sorin, C. et al. Auxin and light control of adventitious rooting in Arabidopsis require ARGONAUTE1. Plant Cell 17, 1343-1359 (2005).

71. Willige, B. C., Isono, E., Richter, R., Zourelidou, M. \& Schwechheimer, C. Gibberellin regulates PIN-FORMED abundance and is required for auxin transportdependent growth and development in Arabidopsis thaliana. Plant Cell 23, 2184-2195 (2011).

72. Ross, J. J., O'Neill, D. P., Smith, J. J., Kerckhoffs, L. H. J. \& Elliott, R. C. Evidence that auxin promotes gibberellin A1 biosynthesis in pea. Plant J. 21, 547 (2000).

73. Han, F. M. \& Zhu, B. G. Evolutionary analysis of three gibberellin oxidase genesin rice, Arabidopsis, and soybean. Gene 473, 23-35 (2011).

74. Fleet, C. M. \& Sun, T. P. A DELLAcate balance: the role of gibberellin in plant morphogenesis. Curr. Opin. Plant. Biol. 8, 77-85 (2005).

75. Ou, C., Jiang, S., Wang, F., Tang, C. \& Hao, N. An RNA-Seq analysis of the pear (Pyrus communis L.) transcriptome, with a focus on genes associated with dwarf. Plant Gene. 4, 69-77 (2015).

76. Silverstone, A. L. et al. Functional analysis of SPINDLY in gibberellin signaling in Arabidopsis. Plant Physiol. 143, 987-1000 (2007).

77. Schomburg, F. M., Bizzell, C. M., Lee, D. J., Zeevaart, J. A. D. \& Amasino, R. M Overexpression of a novel class of gibberellin 2-oxidases decreases gibberellin levels and creates dwarf plants. Plant Cell. 15, 151-163 (2003).

78. Huang, J. et al. Activation of gibberellin 2-oxidase 6 decreases active gibberellin levels and creates a dominant semi-dwarf phenotype in rice (Oryza sativa L.). J. Genet. Genom. 37, 23-36 (2010).

79. Gou, J. Q. et al. Tissue-specific expression of Populus C-19 GA 2-oxidases differentially regulate above- and below-ground biomass growth through control of bioactive GA concentrations. New Phytol. 192, 626-639 (2011).

80. Sponsel, V. M. \& Hedden, P. Gibberellin Biosynthesis and Inactivation. (Springer, Netherlands, 2010).

81. Cowling, R. J., Kamiya, Y., Seto, H. \& Harberd, N. P. Gibberellin dose-response regulation of GA4 gene transcript levels in Arabidopsis. Plant Physiol. 117, 1195-1203 (1998)

82. $\mathrm{Xu}$, Y. L. et al. The Ga5 locus of arabidopsis-thaliana encodes a multifunctional gibberellin 20-oxidase - molecular-cloning and functional expression. Proc Natl Acad. Sci. USA 92, 6640-6644 (1995).

83. Ding, B., Itaya, A. \& Qi, Y. J. Symplasmic protein and RNA traffic: regulatory points and regulatory factors. Curr. Opin. Plant. Biol. 6, 596-602 (2003).

84. Mallory, A. C., Mlotshwa, S., Bowman, L. H. \& Vance, V. B. The capacity of transgenic tobacco to send a systemic RNA silencing signal depends on the nature of the inducing transgene locus. Plant J. 35, 82-92 (2003).

85. Yang, Y. Z. et al. Messenger RNA exchange between scions and rootstocks in grafted grapevines. BMC Plant Biol. 15, 251 (2015)

86. KATO, J. Nonpolar transport of gibberellin through pea stem and a method for its determination. Science 128, 1008-1009 (1958).

87. Liu, Z. H. et al. Cotton GASL genes encoding putative gibberellin-regulated proteins are involved in response to GA signaling in fiber development. Mol. Biol. Rep. 40, 4561-4570 (2013). 